\title{
Aerodynamic Shape Optimization of Wing and Wing-Body Configurations Using Control Theory
}

\author{
James Reuther and Antony Jameson
}

The Research Institute of Advanced Computer Science is operated by Universities Space Research Association, The American City Building, Suite 212, Columbia, MD 21044, (410) 730-2656

Work reported herein was sponsored by NASA under contract NAS 2-13721 between NASA and the Universities Space Research Association (USRA). 



\title{
Aerodynamic Shape Optimization of Wing and Wing-Body Configurations Using Control Theory
}

\author{
J. Reuther \\ Research Institute for Advanced Computer Science \\ Mail Stop T20G-5 \\ NASA Ames Research Center \\ Moffett Field, Califonia 94035, U.S.A. \\ and \\ A. Jameson ${ }^{\dagger}$ \\ Department of Mechanical and Aerospace Engineering \\ Princeton University \\ Princeton, New Jersey 08544, U.S.A.
}

\begin{abstract}
$\underline{\text { Abstract }}$
This paper describes the implementation of optimization techniques based on control theory for wing and wing-body design. In previous studies $[18,19,22]$ it was shown that control theory could be used to devise an effective optimization procedure for airfoils and wings in which the shape and the surrounding body-fitted mesh are both generated analytically, and the control is the mapping function. Recently, the method has been implemented for both potential flows and flows governed by the Euler equations using an alternative formulation which employs numerically generated grids, so that it can more easily be extended to treat general configurations $[34,23]$. Here results are presented both for the optimization of a swept wing using an analytic mapping, and for the optimization of wing and wing-body configurations using a general mesh.
\end{abstract}

\section{Nomenclature}

a spanwise scale factor

$A_{\text {t }}$ Cartesian flux Jacobians

$\mathcal{A}, \mathcal{B}, \mathcal{C}$ metric terms

$b$ design variable

$\hat{b}$ bandwidth of full Jacobian matrix

$B$ generic boundary of flowfield domain

$B_{W} \quad$ wing surface boundary of flowfield domain

$B_{F}$ far-field boundary of flowfield domain

- Student Member AIA A

† James S. McDonnell Distinguished University Professor of Aerospace Engineering. A IAA Fellow
$B_{S}$ symmetry plane boundary of flowfield domain

$C$ transformed flux Jacobians

$C_{i}$ coefficient of $\mathrm{drag}$

$C_{L}$ coefficient of lift

$C_{p}$ coefficient of pressure

$C_{p}^{*}$ coefficient of pressure for sonic flow

$D$ flowfield domain

$E$ total energy

$f_{i}$ components of Cartesian fluxes

$\bar{f}_{1}, f_{1}$ without pressure terms

$F_{1}$ components of transformed fluxes

$F_{\mathrm{i}} \quad F_{\mathrm{i}}$ without pressure terms

$\mathcal{F}$ control law, physical location of boundary

Gis $J h_{i j}^{-1}$

$\mathcal{G}$ gradient of design space

$\overline{\mathcal{G}}$ projected $\mathcal{G}$ into admissible space

$H$ total enthalpy

$I$ cost function

$J$ Jacobian of generalized transformation

$H$ grid transformation matrix

$K$. $K$ at the profile surface

$H_{i}$ grid transformation matrix

$m$ number of design points

$M$ local Mach number

$M_{\infty}$ Mach number at infinity

$n$ number of design variables

$\bar{n}$ number of grid points

$\bar{n}$. components of a unit vector normal

$p$ pressure

$p_{d}$ desired pressure

$\mathcal{P}, \mathcal{Q}, \mathcal{R}$ metric terms

$q$ speed

$q_{\infty}$ speed at infinity

$R$ generic governing equation for flowfield

$S$ normalized arc length 


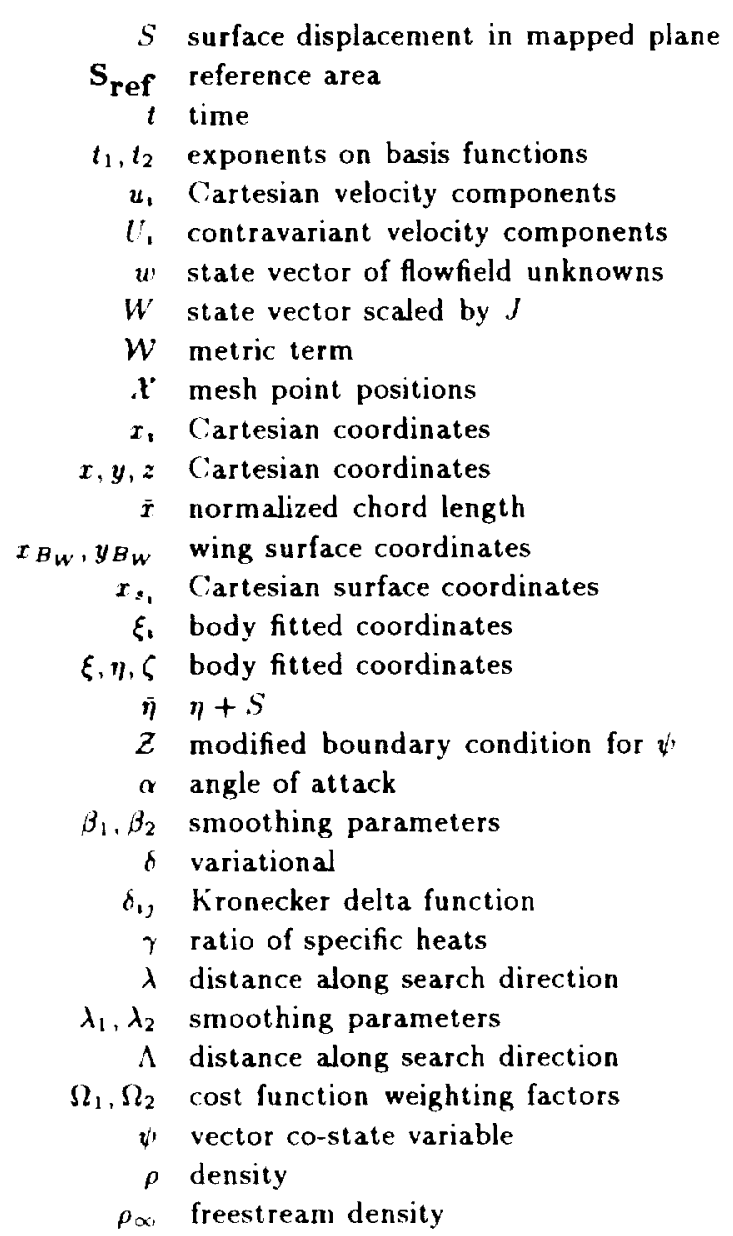

\section{Introduction}

Since about 1960 , there has been rapid progress in the field of Computational Fluid Dynamics (CFD). Especially in the last decade, with substantial improvements in both computer performance and numerical methods, ( :FD has been extensively used together with experimental methods to aid in the aerodynamic design process. While much research continues in the CFD field, accurate and robust solutions are now routinely obtained over complete aircraft configurations for many flow conditions. Modern aircraft designers hope to benefit from this capacity both to refine existing designs at transonic conditions and to develop new designs at supersonic conditions. These highly nonlinear flow regimes require a design fidelity for which only CFD may provide the answers within practical time constraints Thus far however, CFD, like wind tunnel testing, has not had much success in direct aerodynamic shape design. Since the inception of CFD, researchers have sought not only accurate aerodynamic prediction methods for given configurations, but also design methods capable of creating new optimum configurations. Yet, while flow analysis can now be carried out over quite complex configurations using the Navier-Stokes equations with a high degree of con- fidence, direct CFD based design is still limited to very simple two-dimensional and three-dimensional configurations, usually without the inclusion of viscous effects. The CFD-based aerodynamic design methods that do exist can be broken down into three basic categories: inverse surface methods, inverse field methods and numerical optimization methods.

Inverse surface methods derive their name from the fact that they invert the goal of the flow analysis algorithm. Instead of obtaining the surface distribution of an aerodynamic quantity, such as pressure, for a given shape, they calculate the shape for a given surface distribution of an aerodynamic quantity. An alternative way to obtain desirable aerodynamic shapes is through the use of field-based inverse design methods. These methods differ from surface specification methods in that they obtain designs based upon objectives, or constraints, imposed not only upon the configuration surface but everywhere in the flow field. For transonic flows a field-based objective can be to limit shock strength or create shock-free designs. Most of these methods are based on potential flow techniques, and few of them have been extended to three-dimensions

The common trait of all inverse methods is their computational efficiency. Typically, transonic inverse methods require the equivalent of $2-10 \mathrm{com}-$ plete flow solutions in order to render a complete design. Since obtaining a few solutions for simple two-dimensional and three-dimensional designs can be done in at most a few hours on modern computers systems, the computational cost of most inverse methods is considered to be minimal. Unfortunately, they suffer from many limitations and difficulties. Their most glaring limitation is that the objective is built directly into the design process and thus cannot be changed to an arbitrary or more appropriate objective function. The user must therefore be highly experienced in order to be able to prescribe surface distributions or choose initial geometries which lead to the desired aerodynamic properties. In addition, surface inverse methods have a tendency to fail because the target surface distribution is not necessarily attainable. In general it must satisfy constraints to permit the existence of the desired solution. On the other hand, field inverse methods typically only allow for the design of a single shock-free design point, and have no means of properly addressing off-design conditions. Furthermore, it is difficult to formulate inverse methods that can satisfy desired aerodynamic and geometric constraints. In essence, inverse methods require designers to have an $a$ priori knowledge of an optimum pressure distribution that satisfies the geometric and aerodynamic constraints. This limited design capability and difficult implementation has, to date, constrained the applicability of inverse methods.

An alternative approach, which avoids some of the 
difficulties of inverse methods, but only at the price of heavy computational expense, is to use numerical optimization methods. The essence of these methods is very simple: a numerical optimization procedure is coupled directly to an existing CFD analysis algorithm. The numerical optimization procedure attempts to extremize a chosen aerodynamic measure of merit which is evaluated by the chosen CFD code. The configuration is systematically modified through user specified design variables. These design variables must be chosen in such a way as to permit the shape of the configuration to change in a manner that allows the design objective to be improved. Most of these optimization procedures require gradient information in addition to evaluations of the objective function. Here, the gradient refers to changes in the objective function with respect to changes in the design variables. The simplest method of obtaining gradient information is by "brute-force" finite differences. In this technique, the gradient components are estimated by independently perturbing each design variable with a finite step, calculating the corresponding value of the objective function using CFD analysis, and forming the ratio of the differences. The gradient is used by the numerical optimization algorithm to calculate a search direction using steepest descent, conjugate gradient, or quasi-Newton techniques. The optimization algorithm then proceeds by estimating the minimum or maximum of the aerodynamic objective function along the search direction using repeated CFD flow analyses. The entire process is repeated until the gradient approaches zero or further improvement in the aerodynamic objective function is impossible.

The use of numerical optimization for transonic aerodynamic shape design was pioneered by Hicks, Murman and Vanderplaats [13]. They applied the method to two-dimensional profile design subject to the potential flow equation. The method was quickly extended to wing design by Hicks and Henne [11, 12]. Recently, in the work of Reuther, Cliff, Hicks and Van Dam, the method has proven to be successful for the design of supersonic wing/body transport configurations through its extension to three-dimensional flows governed by the Euler equations [33]. In all of these cases brute-force finite difference methods were used to obtain the required gradient information.

These methods are very versatile, allowing any reasonable aerodynamic quantity to be used as the objective function. They can be used to mimic an inverse method by minimizing the difference between target and actual pressure distributions, or may instead be used to maximize other aerodynamic quantities of merit such as $L / D$. Geometric constraints can be readily enforced by a proper choice of design variables. Aerodynamic constraints can be treated either by adding weighted terms to the objective function or by the use of a constrained optimization algorithm. Unfortunately, these brute-force numerical optimization methods, unlike the inverse methods, are computationally expensive because of the large number of flow solutions needed to determine the gradient information for a useful number of design variables. For three-dimensional configurations. hundreds or even thousands of design variables may be necessary. This implies that tens of thousands of flow analyses would be required for a complete design.

\section{Formulation of the design problem as a control problem}

Clearly, alternative methods must be developed which have the flexibility and power of current numerical optimization codes but do not require such large computational resources. These new methods must avoid the limitations and difficulties of traditional inverse methods while approaching their inherent computational efficiency.

One means of attaining such a design method is by treating the design problem within the mathematical theory for control of systems governed by partial differential equations [29]. Suppose that the boundary is defined by a function $\mathcal{F}(b)$, where $b$ is the position vector of the design variables, and the desired objective is measured by a cost function $I$. This may, for example, measure the deviation from a desired surface pressure distribution, but it can also represent other measures of performance such as the drag or the lift/drag ratio. Suppose that a variation $\delta \mathcal{F}$ in the control produces a variation $\delta I$ in the cost. $\delta I$ can be expressed to first order as an inner product

$$
\delta I=(\mathcal{G}, \delta \mathcal{F})
$$

where the gradient, $\mathcal{G}$, of the cost function with respect to the control, is independent of the particular variation $\delta \mathcal{F}$. Following control theory $\mathcal{G}$ can be determined by solving an adjoint equation. If one makes a shape change

$$
\delta \mathcal{F}=-\lambda \mathcal{G}
$$

where $\lambda$ is sufficiently small and positive, then

$$
\delta I=-\lambda(\mathcal{G}, \mathcal{G})<0
$$

assuring a reduction in $I$. The method can be accelerated by choosing $\delta \mathcal{F}$ not simply as a multiple of the gradient (steepest descent) but instead as a more sophisticated search direction provided by numerical optimization.

For flow about an airfoil or wing, the aerodynamic properties which define the cost function are functions of the flow-field variables $(w)$, the physical locations of the mesh points within the volume $(\boldsymbol{r})$, 
and the physical location of the boundary $(\mathcal{F})$. Then

$$
I=I\left(w, \mathcal{X}^{\prime}, \mathcal{F}\right),
$$

and a change in $\mathcal{F}$ results in a change

$$
\delta I=\frac{\partial I^{T}}{\partial w} \delta w+\frac{\partial I^{T}}{\partial \mathcal{X}} \delta \mathcal{X}+\frac{\partial I^{T}}{\partial \mathcal{F}} \delta \mathcal{F},
$$

in the cost function. As pointed out by Baysal and Eleshaky [4] each term in (2), except for $\delta w$, can be easily obtained. $\frac{\partial I}{\partial w}, \frac{\partial I}{\partial \mathcal{X}^{\prime}}$ and $\frac{\partial I}{\partial \mathcal{F}}$ can be obtained directly without a flowfield evaluation since they are partial derivatives. $\delta \mathcal{F}$ is simply the surface modification and $\delta \mathcal{X}$ can be determined by either working out the exact analytical values from a mapping, or by successive grid generation for each design variable, so long as this cost is significantly less then the cost of the flow solution. For solutions requiring a large number of mesh points where grid generation becomes expensive, an alternative method for calculating $\delta \mathcal{X}^{\prime}$ can be formulated using grid perturbation. Brute-force methods evaluate the gradient by making a small change in each design variable separately, and then recalculate both the grid and flow-field variables. This requires a number of additional flow calculations equal to the number of design variables. Using control theory, the governing equations of the flowfield are introduced as a constraint in such a way that the final expression for the gradient does not require multiple flow solutions. In order to achieve this result, $\delta w$ must be eliminated from (2). The governing equation $R$ expresses the dependence of $w, \boldsymbol{x}$ and $\mathcal{F}$ within the flowfield domain $D$.

$$
R(w, \boldsymbol{x}, \mathcal{F})=0,
$$

Thus $\delta w$ is determined from the equation

$$
\delta R=\left[\frac{\partial R}{\partial w}\right] \delta w+\left[\frac{\partial R}{\partial \mathcal{X}^{\prime}}\right] \delta \mathcal{X}+\left[\frac{\partial R}{\partial \mathcal{F}}\right] \delta \mathcal{F}=0 .
$$

Next, introducing a Lagrange Multiplier $\psi$, we have

$$
\begin{aligned}
\delta I= & \frac{\partial I^{T}}{\partial w} \delta w+\frac{\partial I^{T}}{\partial \mathcal{X}} \delta \mathcal{X}+\frac{\partial I^{T}}{\partial \mathcal{F}} \delta \mathcal{F} \\
& -\psi^{T}\left(\left[\frac{\partial R}{\partial w}\right] \delta w+\left[\frac{\partial R}{\partial \mathcal{X}^{T}}\right] \delta \mathcal{X}+\left[\frac{\partial R}{\partial \mathcal{F}}\right] \delta \mathcal{F}\right) \\
= & \left\{\frac{\partial I^{T}}{\partial w}-\psi^{T}\left[\frac{\partial R}{\partial w}\right]\right\} \delta w \\
& +\left\{\frac{\partial I^{T}}{\partial \mathcal{X}^{T}}-\psi^{T}\left[\frac{\partial R}{\partial \mathcal{X}^{\prime}}\right]\right\} \delta \mathcal{X} \\
& +\left\{\frac{\partial I^{T}}{\partial \mathcal{F}}-\psi^{T}\left[\frac{\partial R}{\partial \mathcal{F}}\right]\right\} \delta \mathcal{F}
\end{aligned}
$$

('hoosing $\psi$ to satisfy the adjoint equation

$$
\left[\frac{\partial R}{\partial u}\right]^{T} \psi=\frac{\partial I}{\partial u}
$$

the first term is eliminated, and we find that

$$
\delta I=\mathcal{G}^{T} \delta \mathcal{F}
$$

where

$$
\mathcal{G}^{T}=\left\{\frac{\partial I^{T}}{\partial \mathcal{X}}-\psi^{T}\left[\frac{\partial R}{\partial \mathcal{X}}\right]\right\} \frac{\delta \mathcal{X}}{\delta \mathcal{F}}+\frac{\partial I^{T}}{\partial \mathcal{F}}-\psi^{T}\left[\frac{\partial R}{\partial \mathcal{F}}\right]
$$

The advantage is that (5) is independent of $\delta w$, with the result that the gradient of $I$ with respect to an arbitrary number of design variables can be determined without the need for additional flow-field evaluations. The main cost is in solving the adjoint equation (4). In general, the adjoint problem is about as complex as a flow solution. However, if the number of design variables is large, the cost differential between one adjoint solution and the large number of flowfield evaluations required to determine the gradient by finite differences becomes compelling.

Once the gradient is calculated by (6) a modification following (1) can then be made. After making such a modification, the gradient can be recalculated and the process repeated to follow a path of steepest descent until a minimum is reached. In order to avoid violating geometric constraints, such as a minimum acceptable wing thickness, the gradient may be projected into the allowable subspace within which the constraints are satisfied. In this way procedures can be devised which must necessarily converge at least to a local minimum. The efficiency may be improved by performing line searches to find the minimum in a search direction defined by the negative gradient, and also by the use of more sophisticated descent methods such as conjugate gradient or quasi-Newton algorithms. There is the possibility of more than one local minimum, but in any case the method will lead to an improvement over the original design. Furthermore, unlike the traditional inverse algorithms, any measure of performance can be used as the cost function.

In this research, the adjoint approach is used to permit a dramatic reduction in the computational cost of each design solution, since the gradient cost will be reduced to the cost of approximately two flow evaluations (provided the adjoint equations are about as computationally expensive as the flow equations) instead of the traditional $n+1$ evaluations, where $n$ is the number of design variables. The key point is that the cost of the optimization method is no longer proportional to the number of design variables, which has been the limiting factor in brute-force aerodynamic optimization methods.

Another significant advantage of this method is its applicability to multi-point design problems. Because of its efficient use of computer resources, two or three design points can be included in the optimization procedure by solving separate adjoint problems for each design point. The resulting procedure 
yields a method which is capable of calculating the combined gradient from all design points from order $2 m$ equivalent flow calculations, with $m$ being the number of design points. Even in the case where there is a large number of design points a significant benefit is still realized when compared with bruteforce calculations where the number of solutions required is $m n$. The use of this approach will allow the performance benefits at various design points to be considered together, thus obtaining a more optimal overall design.

A variety of alternative formulations of the design problem can then be treated systematically within the framework of the mathematical theory for control of systems governed by partial differential equations [29]. The use of this method for aerodynamic design was first introduced by Jameson [18] who examined its application to transonic flow governed by both the potential flow and Euler equations [19, 22]. In his work, control theory was applied directly to the partial differential equations governing the flow solution. Thus the adjoint equations were formed as a system of differential equations. These adjoint differential equations were then discretized and solved in the same manner as the flow equations to obtain the necessary gradient information. This approach, often termed the continuous sensitivity analysis, was used by Jameson in conjunction with analytic grid mappings, to formulate directly at the differential level, the necessary systems of equations defined by equation (6).

Steps $(2-6)$ may alternatively be applied to the discrete equations which approximate the governing differential equations. This approach (discrete sensitivity analysis) is now gaining favor in the work of Korovi, Newman, Taylor, Hou and Jones $[30,26,15,24]$ and also Baysal, Eleshaky and Burgreen $[3,4,5,7,2,8,9,6]$. The continuous and the discrete formulations methods can be very similar, depending upon the discretization of (4). The method used by Jameson has an advantage in that the discretization and iteration scheme used to solve the flowfield system can also be applied directly to the adjoint system. Therefore, the robust iteration algorithms and convergence acceleration techniques that have been matured for CFD algorithms can be directly ported for the solution of the adjoint system. Discrete sensitivity methods for equations (2 - 6) often resort to matrix elimination methods to solve (4). While direct techniques to solve these large sparse systems can be robust and reliable they suffer when the number of points becomes large because the operation count grows as $O\left(\hat{n} \hat{b}^{2}\right)$ and the storage goes as $O(\dot{n} \dot{b})$, where $\hat{n}$ is the number of unknowns and $\dot{b}$ is the bandwidth. Therefore, in order to solve these large systems, alternatives such as sophisticated matrix decomposition [28] or incremental iterative methods [25] must be employed.
However, these alternatives, whether applied to the flow solution system or, as here, to the adjoint system, have not proven to be as efficient as methods developed for CFD. CFD approaches altogether avoid the direct matrix elimination process by relaxing the system either point-by-point or line-byline in a pseudo-time procedure. When the number of mesh points becomes large, especially in the case of three-dimensional problems, the $O(\hat{n})$ operational counts and the $O(\hat{n})$ storage of explicit iteration schemes used in CFD can significantly reduce the time and memory required to solve the adjoint system. Methods that use matrix decomposition or the Generalized Minimum Residual (GMRES) iterative approach to solve the discrete sensitivity problem have shown modest improvements over standard Gaussian elimination [28] and [37, 14]. Nevertheless, these methods are currently still not competitive in terms of both time and memory with mature CFDlike algorithms. The efficiency of discrete sensitivity methods may be improved by the introduction of incremental iterative strategies for solving the matrix elimination problem. Present work by Taylor et al. [25] to develop such methods shows great promise. Currently the use of continuous sensitivity analysis eliminates the need for developing incremental strategies since the existing (and presumably mature) flow solution algorithm can be used directly for the adjoint solution.

Other applications of control theory in aerodynamics have been explored by Pironneau for optimum shape design of systems governed by elliptic equations [31]. More recently Ta'asan, Kuruvila, and Salas implemented a one shot approach in which the constraint represented by the flow equations is only required to be satisfied by the final converged solution $[36,27]$. Pironneau has also studied the use of control theory for optimum shape design of systems governed by the Navier-Stokes equations [32].

\section{Three-Dimensional Design Using the Euler Equations}

The application of control theory to aerodynamic design problems is illustrated by treating the case of three-dimensional wing design, using the inviscid Euler equations as the mathematical model for compressible flow. In this case it proves convenient to denote the Cartesian coordinates and velocity components by $x_{1}, x_{2}, x_{3}$ and $u_{1}, u_{2}, u_{3}$, and to use the convention that summation over $i=1$ to 3 is implied by a repeated index $i$. The three-dimensional Euler equations may be written as

$$
\frac{\partial w}{\partial t}+\frac{\partial f_{i}}{\partial x_{i}}=0 \text { in } D
$$


where

$$
u=\left\{\begin{array}{c}
\rho \\
\rho u_{1} \\
\rho u_{2} \\
\rho u_{3} \\
\rho E
\end{array}\right\}, \quad f_{i}=\left\{\begin{array}{c}
\rho u_{i} \\
\rho u_{i} u_{1}+p \delta_{i 1} \\
\rho u_{i} u_{2}+p \delta_{i 2} \\
\rho u_{i} u_{3}+p \delta_{i 3} \\
\rho u_{i} H
\end{array}\right\}
$$

and $\delta_{i j}$ is the Kronecker delta function. Also,

$$
p=(\gamma-1) \rho\left\{E-\frac{1}{2}\left(u_{i}^{2}\right)\right\}
$$

and

$$
\rho H=\rho E+p
$$

where $\gamma$ is the ratio of the specific heats. Consider a transformation to coordinates $\xi_{1}, \xi_{2}, \xi_{3}$ where

$$
k_{i j}=\left[\frac{\partial x_{i}}{\partial \xi_{j}}\right], \quad J=\operatorname{det}(H), \quad k_{i j}^{-1}=\left[\frac{\partial \xi_{i}}{\partial x_{j}}\right]
$$

Introduce contravariant velocity components as

$$
\left\{\begin{array}{l}
U_{1} \\
J_{2} \\
U_{3}
\end{array}\right\}=K^{-1}\left\{\begin{array}{l}
u_{1} \\
u_{2} \\
u_{3}
\end{array}\right\}
$$

The Euler equations can now be written as

$$
\frac{\partial W}{\partial t}+\frac{\partial F_{i}}{\partial \xi_{i}}=0 \text { in } D
$$

with

$$
W=J\left\{\begin{array}{c}
\rho \\
\rho u_{1} \\
\rho u_{2} \\
\rho u_{3} \\
\rho E
\end{array}\right\}, F_{i}=J\left\{\begin{array}{c}
\rho U_{i} \\
\rho U_{i} u_{1}+\frac{\partial \xi_{1}}{\partial x_{1}} p \\
\rho U_{i} u_{2}+\frac{\partial \xi_{1}}{\partial x_{2}} p \\
\rho U_{i} u_{3}+\frac{\partial \xi_{1}}{\partial x_{3}} p \\
\rho U_{i} H
\end{array}\right\}
$$

Assume now that designs are limited to wing and wing-body configurations using a $\mathrm{C}-\mathrm{H}$ topology mesh. If a body is present it is assumed to exist as part of the face containing the symmetry plane. Thus the new computational coordinate system conforms to the wing in such a way that the wing surface $B_{W}$ is represented by $\xi_{2}=0$ and the body and symmetry plane $B_{S}$ conform such that $\xi_{3}=0$. Then the flow is determined as the steady state solution of equation (11) subject to the flow tangency conditions

$$
\begin{array}{ll}
U_{2}=0 & \text { on } B_{\boldsymbol{W}} \\
U_{3}=0 & \text { on } B_{\boldsymbol{S}} .
\end{array}
$$

At the far field boundary $B_{F}$, freestream conditions are specified for incoming waves, as in the twodimensional case, while outgoing waves are determined by the solution.
As the first example, suppose that it is desired to control the surface pressure by varying the wing shape. It is convenient to retain a fixed computational domain. Variations in the shape then result in corresponding variations in the mapping derivatives defined by $K$. Introduce the cost function

$$
I=\frac{1}{2} \iint_{B_{W}}\left(p-p_{d}\right)^{2} d \xi_{1} d \xi_{3}
$$

where $p_{d}$ is the desired pressure on the wing. The design problem is now treated as a control problem where the control function is the wing shape, which is to be chosen to minimize I subject to the constraints defined by the flow equations (7-12). A variation in the shape will cause a variation $\delta p$ in the pressure and consequently a variation in the cost function

$$
\delta I=\iint_{B_{W^{\prime}}}\left(p-p_{d}\right) \delta p d \xi_{1} d \xi_{3}
$$

Since $p$ depends on $w$ through the equation of state $(9-10)$, the variation $\delta p$ can be determined from the variation $\delta w$. Define the Jacobian matrices

$$
A_{i}=\frac{\partial f_{i}}{\partial w}, \quad C_{i}=J K_{i j}^{-1} A_{j} .
$$

Then the equation for $\delta w$ in the steady state becomes

$$
\frac{\partial}{\partial \xi_{i}}\left(\delta F_{i}\right)=0
$$

where

$$
\delta F_{i}=C_{i} \delta w+\delta\left(J \frac{\partial \xi_{i}}{\partial x_{j}}\right) f_{j}
$$

Now, multiplying by a vector co-state variable $\psi$ and integrating over the domain

$$
\int_{D}{ }^{T}\left(\frac{\partial \delta F_{i}}{\partial \xi_{i}}\right) d \xi_{j}=0
$$

and if $\psi$ is differentiable this may be integrated by parts to give

$$
\int_{D}\left(\frac{\partial \psi^{T}}{\partial \xi_{i}} \delta F_{i}\right) d \xi_{j}=\int_{B}\left(\bar{n}_{i} \psi^{T} \delta F_{i}\right) d \xi_{j}
$$

where $\hat{n}_{i}$ are components of a unit vector normal to the boundary. Thus the variation in the cost function may now be written

$$
\begin{aligned}
\delta I= & \iint_{B_{w}}\left(p-p_{d}\right) \delta p d \xi_{1} d \xi_{3} \\
& -\int_{D}\left(\frac{\partial \psi^{T}}{\partial \xi_{i}} \delta F_{i}\right) d \xi_{j} \\
& +\int_{B}\left(\bar{n}_{i} \psi^{T} \delta F_{i}\right) d \xi_{j}
\end{aligned}
$$

On the wing surface $B_{W}, \bar{n}_{1}=\bar{n}_{3}=0$ and on the body and symmetry plane $B_{S}, \bar{n}_{1}=\bar{n}_{2}=0$. It 


$$
\begin{aligned}
& \delta F_{2}=J\left\{\begin{array}{c}
0 \\
\frac{\partial \xi_{2}}{\partial x_{1}} \delta p \\
\frac{\partial \xi_{2}}{\partial x_{2}} \delta p \\
\frac{\partial \xi_{2}}{\partial x_{3}} \delta p \\
0
\end{array}\right\}+p\left\{\begin{array}{c}
0 \\
\delta\left(J \frac{\partial \xi_{2}}{\partial x_{1}}\right) \\
\delta\left(J \frac{\partial \xi_{2}}{\partial x_{2}}\right) \\
\delta\left(J \frac{\partial \xi_{3}}{\partial x_{3}}\right) \\
0
\end{array}\right\} \text { on } B_{W} \\
& \delta F_{3}=J\left\{\begin{array}{c}
0 \\
0 \\
\frac{\partial \xi_{3}}{\partial x_{1}} \delta p \\
\frac{\partial \xi_{3}}{\partial x_{2}} \delta p \\
\frac{\partial \xi_{3}}{\partial x_{3}} \delta p \\
0
\end{array}\right\}+p\left\{\begin{array}{c}
\delta\left(J \frac{\partial \xi_{3}}{\partial x_{1}}\right) \\
\delta\left(J \frac{\partial \xi_{3}}{\partial x_{2}}\right) \\
\delta\left(J \frac{\partial \xi_{3}}{\partial x_{3}}\right) \\
0
\end{array}\right\} \text { on } B_{S} .
\end{aligned}
$$

Suppose now that $\psi$ is the steady state solution of the adjoint equation

$$
\frac{\partial \psi}{\partial t}-C_{i}^{T} \frac{\partial \psi}{\partial \xi_{i}}=0 \text { in } D
$$

At the outer boundary incoming characteristics for $\psi$ ' correspond to outgoing characteristics for $\delta w$. Consequently, as in the two-dimensional case, one can choose boundary conditions for $v$ such that

$$
\bar{n}_{i} \psi^{T} C_{2} \delta u=0 .
$$

Then if the coordinate transformation is such that $\delta\left(J \mathrm{~K}^{-1}\right)$ is negligible in the far field, the only remaining boundary terms are

$$
\begin{aligned}
& -\iint_{B_{w}} \psi^{T} \delta F_{2} d \xi_{1} d \xi_{3} \\
& -\iint_{B_{S}}{ }^{T}{ }^{T} \delta F_{3} d \xi_{1} d \xi_{2} .
\end{aligned}
$$

Thus by letting $\psi$ satisfy the boundary conditions,

$$
\begin{aligned}
& J\left(\psi_{2} \frac{\partial \xi_{2}}{\partial x_{1}}+\psi_{3} \frac{\partial \xi_{2}}{\partial x_{2}}+\psi_{4} \frac{\partial \xi_{2}}{\partial x_{3}}\right)=\left(p-p_{d}\right) \text { on } B w \\
& J\left(\psi_{2} \frac{\partial \xi_{3}}{\partial x_{1}}+\psi_{3} \frac{\partial \xi_{3}}{\partial x_{2}}+\psi_{4} \frac{\partial \xi_{3}}{\partial x_{3}}\right)=0 \text { on } B_{S}, \quad \text { (19) }
\end{aligned}
$$

we find finally that

$$
\begin{aligned}
& \delta t=-\int_{D} \frac{\partial v^{T}}{\partial \varepsilon_{1}} \cdot\left(j \frac{\partial \varepsilon_{1}}{\partial x_{3}}\right) s, d \varepsilon_{k} \\
& -\iint_{B_{W}}\left\{v^{\prime} \delta\left(j \frac{\partial \varepsilon_{2}}{\partial x_{1}}\right)+v_{3} \cdot\left(J \frac{\partial \varepsilon_{2}}{\partial x_{2}}\right)+v_{4} \delta\left(j \frac{\partial \xi_{2}}{\theta x_{3}}\right)\right\} p d \varepsilon_{1} d \varepsilon_{3}
\end{aligned}
$$

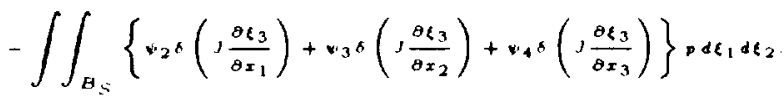

Now

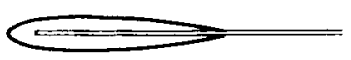

1a: $x, y$-Plane.

1b: $\xi, \eta$-Plane

Figure 1: Sheared Parabolic Mapping.

\section{Wing Design Using Analytic Mesh Generation}

A convenient way to treat a wing is to introduce sheared parabolic coordinates as shown in figure 1 through the transformation

$$
\begin{aligned}
x & =x_{0}(\zeta)+\frac{1}{2} a(\zeta)\left\{\xi^{2}-(\eta+S(\xi, \zeta))^{2}\right\} \\
y & =y_{0}(\zeta)+a(\zeta) \xi(\eta+S(\xi, \zeta)) \\
z & =\zeta .
\end{aligned}
$$

Here $x=x_{1}, y=x_{2}, z=x_{3}$ are the Cartesian coordinates, and $\xi$ and $\eta+. S$ correspond to parabolic coordinates generated by the mapping

$$
x+i y=x_{0}+i y_{0}+\frac{1}{2} a(\zeta)\{\xi+i \eta\}^{2}
$$

at a fixed span station $\zeta$ and $\bar{\eta}=\eta+S \cdot x_{0}(\zeta)$ and $y_{0}(\zeta)$ are the coordinates of a singular line which is swept to lie just inside the leading edge of a swept wing, while $a(\zeta)$ is a scale factor to allow for spanwise chord variations. The surface $\eta=0$ is a shallow bump corresponding to the wing surface, with a height $S(\xi, \zeta)$ determined by the equation

$$
\xi+i S=\sqrt{2\left(x_{B_{W}}+i y_{B_{W}}\right)},
$$

where $x_{B_{W}}(z)$ and $y_{B_{W}}(z)$ are coordinates of points lying on the wing surface. We now treat $S(\xi, \zeta)$ as the control.

In this case the transformation matrix $\frac{\partial x_{1}}{\partial \xi}$, becomes

$$
\begin{aligned}
\mathscr{K} & =\left[\begin{array}{ccc}
a\left(\xi-\eta S_{\xi}\right) & -a \bar{\eta} & \mathcal{A}-a \bar{\eta} S_{\zeta} \\
a\left(\bar{\eta}+\xi S_{\xi}\right) & a \xi & \mathcal{B}+a \xi S_{\zeta} \\
0 & 0 & 1
\end{array}\right] \\
& =\left[\begin{array}{ccc}
x_{\xi} & x_{\eta} & \mathcal{A}+x_{\eta} S_{\zeta} \\
y_{\xi} & y_{\eta} & \mathcal{B}+y_{\eta} S_{\zeta} \\
0 & 0 & 1
\end{array}\right]
\end{aligned}
$$

where

$$
\mathcal{A}=a_{\zeta} \frac{x-x_{0}}{a}+x_{0_{\zeta}}, \quad \mathcal{B}=a_{\zeta} \frac{y-y_{0}}{a}+y_{0_{\zeta}} .
$$

$$
J=x_{\xi} y_{\eta}-x_{\eta} y_{\xi}=\xi^{2}+\bar{\eta}^{2}
$$


and

$$
J K^{-1}=\left[\begin{array}{ccc}
y_{\eta} & -x_{\eta} & x_{\eta} \mathcal{B}-y_{\eta} \mathcal{A} \\
-y_{\xi} & x_{\xi} & y_{\xi} \mathcal{A}-x_{\xi} \mathcal{B}-J S_{\zeta} \\
0 & 0 & J
\end{array}\right]
$$

Then under a modification $\delta S$

$$
\begin{aligned}
\delta x_{\xi} & =-a\left(\delta S S_{\xi}+\bar{\eta} \delta S_{\xi}\right) \\
\delta x_{\eta} & =-a \delta S \\
\delta y_{\xi} & =a\left(\delta S+\xi \delta S_{\xi}\right) \\
\delta y_{\eta} & =0 .
\end{aligned}
$$

Thus

$$
\delta J=2 a^{2} \bar{\eta} \delta S
$$

and

$$
\delta\left(J K^{-1}\right)=\left[\begin{array}{c|c|c}
0 & a \delta S & -a B \delta S \\
-\delta y_{\xi} & \delta x_{\xi} & \mathcal{W} \\
0 & 0 & \delta J
\end{array}\right] .
$$

where

$$
\mathcal{W}=\delta y_{\xi} \mathcal{A}-\delta x_{\xi} \mathcal{B}-a_{\zeta} \frac{J}{a} \delta S-\delta J S_{\zeta}-J \delta S_{z}
$$

Inserting these formulas in equation (20) we find that the volume integral in $\delta I$ is

$$
\begin{aligned}
& \iiint \frac{\partial \psi^{T}}{\partial \xi} \delta S f_{2} d \xi d \eta d \zeta \\
& \quad-\iiint \frac{\partial \psi^{T}}{\partial \eta}\left\{-\delta y_{\xi} f_{1}+\delta x_{\xi} f_{2}+\mathcal{W} f_{3}\right\} d \xi d \eta d \zeta \\
& \quad+\iiint \frac{\partial \psi^{T}}{\partial \zeta} \delta J f_{3} d \xi d \eta d \zeta,
\end{aligned}
$$

where $\delta$ and $\delta S$ are independent of $\eta$. Therefore, integrating over $\%$, the variation in the cost function can be reduced to a surface integral of the form

$\delta I=\iint_{B_{\mathfrak{W}}}\left(\mathcal{P}(\xi, \zeta) \delta S-\mathcal{Q}(\xi, \zeta) \delta S_{\xi}-\mathcal{R}(\xi, \zeta) \delta S_{\zeta}\right) d \xi d \zeta$

Here

$$
\begin{aligned}
\mathcal{P}= & a\left(\psi_{2}+S_{\xi} \psi_{3}+\mathcal{C} \psi_{4}\right) p \\
& -\int \frac{\partial \psi^{T}}{\partial \xi}\left\{\xi f_{1}+\eta f_{2}+(\xi \mathcal{A}+\bar{\eta} \mathcal{B}) f_{3}\right\} d \eta \\
& -\int \frac{\partial \psi^{T}}{\partial \eta}\left(f_{1}+\dot{\zeta}_{\xi} f_{2}+\mathcal{C} f_{3}\right) d \eta \\
& -\int \frac{\partial \psi^{T}}{\partial \zeta} J d \eta \\
\mathcal{Q}= & a\left(\xi \psi_{2}+\bar{\eta} \psi_{3}\right) p \\
& +\int \frac{\partial \psi^{T}}{\partial \eta}\left\{\xi f_{1}+\eta f_{2}+(\xi \mathcal{A}+\bar{\eta} \mathcal{B}) f_{3}\right\} d \eta \\
\mathcal{R}= & J \psi_{4} p \\
& +\int \frac{\partial f_{3}}{\partial \eta} J \psi_{4} d \eta,
\end{aligned}
$$

where

$$
\mathcal{C}=2 a \bar{\eta} S_{\zeta}-\mathcal{A}-\mathcal{B} S_{\xi}+\frac{J}{a} .
$$

Also the shape change will be confined to a boundary region of the $\xi-\zeta$ plane, so we can integrate by parts to obtain

$$
\delta I=\iint_{B_{W}}\left(\mathcal{P}+\frac{\partial \mathcal{Q}}{\partial \xi}+\frac{\partial \mathcal{R}}{\partial \zeta}\right) \delta S d \xi d \zeta .
$$

Thus to reduce I we can choose

$$
\delta S=-\lambda\left(\mathcal{P}+\frac{\partial \mathcal{Q}}{\partial \xi}+\frac{\partial \mathcal{R}}{\partial \zeta}\right)
$$

where $\lambda$ is sufficiently small and non-negative.

In order to impose a thickness constraint we can define a baseline surface $S_{0}(\xi, \zeta)$ below which $S(\xi, \zeta)$ is not allowed to fall. Now if we take $\lambda=\lambda(\xi, \zeta)$ as a non-negative function such that

$$
S(\xi, \zeta)+\delta S(\xi, \zeta) \geq S_{0}(\xi, \zeta) .
$$

Then the constraint is satisfied, while

$$
\delta I=-\iint_{B_{\mathcal{W}}} \lambda\left(\mathcal{P}+\frac{\partial \mathcal{Q}}{\partial \xi}+\frac{\partial \mathcal{R}}{\partial \zeta}\right)^{2} d \xi d \zeta \leq 0
$$

or

$$
\mathcal{G}=\mathcal{P}+\frac{\partial \mathcal{Q}}{\partial \xi}+\frac{\partial \mathcal{R}}{\partial \zeta}
$$

To make sure that no discontinuities are introduced in the modified shape, smoothing can be introduced in the update process by setting

$$
\beta_{1} \delta \mathcal{Z}-\frac{\partial}{\partial \xi} \beta_{2} \frac{\partial}{\partial \xi} \delta \mathcal{Z}=-\left(\mathcal{P}+\frac{\partial \mathcal{Q}}{\partial \xi}+\frac{\partial \mathcal{R}}{\partial \zeta}\right)
$$

Then, integrating by parts in the $\xi$ direction gives

$$
\begin{aligned}
\delta l & =-\iint_{B_{W}}\left(\beta_{1} \delta \mathcal{Z}-\frac{\partial}{\partial \xi} \beta_{2} \frac{\partial}{\partial \xi} \delta \mathcal{Z}\right) \delta \mathcal{Z} d \xi d \eta \\
& =-\iint_{B_{W}}\left(\beta_{1} \delta \mathcal{Z}^{2}+\frac{\partial}{\partial \xi} \beta_{2}\left(\frac{\partial}{\partial \xi} \delta \mathcal{Z}\right)^{2}\right) d \xi d \eta
\end{aligned}
$$

\section{Wing-Body Design Using a General Mesh}

In order to treat a more general mesh we revert to equations (14-20). The difficulty in using these equations is that the variation of the metric terms in the equations needs to be obtained in order to construct $\delta I$ in equation (20). One way to accomplish this is to use finite differences to calculate the necessary information. While this approach would avoid the use of multiple flow solutions to determine the gradient, it would unfortunately still require the mesh generator to be used repeatedly. The number of mesh solutions required would be proportional to 
the number of design variables. This may be acceptable, since the flow solution process is typically much more computationally expensive than grid generation. Such a method should then ensure a significant savings over using finite differences for both the grid generation and flow solution processes. However, for three-dimensional designs where both the number of design variables and the computational cost of grid generation can be high, this method is excessively expensive. Further, for complicated threedimensional configurations, for which it is still not practical to integrate fully automatic grid generation into the solution process, the method will not be feasible.

This motivates the need to find a method which by-passes these difficulties. In order to remove the cost of the successive grid generation from the gradient calculation, a successive grid perturbation method is therefore used. In this approach, which was also used by Burgreen and Baysal [7], an initial structured curvilinear body-fitted grid over the initial configuration is created by any grid generation process before optimization. Then the geometry as well as the grid become inputs to the optimization process. New grids, which conform to the surface as it is modified, can then be generated by shifting the grid points along each grid index line projecting from the surface by an amount which is attenuated as the arc length from the surface increases. If the outer boundary of the grid domain is held constant the modification to the grid has the form

$$
x_{i}^{\text {new }}=x_{i}^{\text {old }}+\mathcal{S}^{\text {old }}\left(x_{s_{i}}^{\text {new }}-x_{s_{i}}^{\text {old }}\right),
$$

where $x_{i}$ represents the volume grid points, $x_{s}$ represents the surface grid points and $S$ represents the arclength along the radial mesh line measured from the outer domain, normalized so that $S=1$ at the inner surface. The required variations in the metric terms can then be obtained in terms of surface perturbations since,

$$
\delta x_{i}=\mathcal{S}^{\text {old }} \delta x_{s_{1}} .
$$

and

$$
\delta \frac{\partial x_{i}}{\partial \xi_{j}}=\mathcal{S}^{o l d} \delta \frac{\partial x_{s_{1}}}{\partial \xi_{j}}
$$

We introduce,

$$
\begin{aligned}
G_{1 \jmath} & =J h_{i j}^{-1} \\
& =\left[\begin{array}{l|l|l}
y_{\eta} z_{\zeta}-y_{\zeta} z_{\eta} & x_{\zeta} z_{\eta}-x_{\eta} z_{\zeta} & x_{\eta} y_{\zeta}-x_{\zeta} y_{\eta} \\
y_{\zeta} z_{\xi}-y_{\xi} z_{\zeta} & x_{\xi} z_{\zeta}-x_{\zeta} z_{\xi} & x_{\zeta} y_{\xi}-x_{\xi} y_{\zeta} \\
y_{\xi} z_{\eta}-y_{\eta} z_{\xi} & x_{\eta} z_{\xi}-x_{\xi} z_{\eta} & x_{\xi} y_{\eta}-x_{\eta} y_{\xi}
\end{array}\right] .
\end{aligned}
$$

Now it is convenient to rewrite equation (20) after integration by parts as

$$
\begin{aligned}
& \delta I=+\int_{D} \psi^{T} \frac{\partial}{\partial \xi_{1}}\left(\delta G_{1}, f,\right) d \xi_{k} \\
& -\iint_{B_{W}}\left\{\psi^{\prime}{ }^{T} \delta G_{2}, \bar{f}_{j}\right\} p d \xi_{1} d \xi_{3}
\end{aligned}
$$

$$
-\iint_{B_{S}}\left\{\psi^{T} \delta G_{3}, \bar{f}_{3}\right\} p d \xi_{1} d \xi_{2}
$$

where $\vec{f}_{j}$ represent the flux components $f_{j}$ with the pressure terms dropped. From the definition of $C_{i j}$ we have, for example,

$$
\begin{aligned}
& \delta G_{11}=\delta\left(y_{\eta}\right) z_{\zeta}+\delta\left(z_{\zeta}\right) y_{\eta}-\delta\left(y_{\zeta}\right) z_{\eta}-\delta\left(z_{\eta}\right) y_{\zeta} \\
& =\mathcal{S}^{\text {old }}\left[\delta\left(y_{\eta_{\diamond}}\right) z_{\zeta}+\delta\left(z_{\zeta}\right) y_{\eta}-\delta\left(y_{\zeta_{\diamond}}\right) z_{\eta}-\delta\left(z_{\eta_{\diamond}}\right) y_{\zeta}\right] .
\end{aligned}
$$

Substituting these expressions into equation (25) allows us to integrate along the index direction projecting from the configuration surface without any dependence on particular design variables, since the metric variations are fully determined by the surface perturbations. Thus, the expression for the variation in the cost function can be reduced to surface integrals only.

While this type of grid perturbation method does not guarantee that grid lines will not eventually cross if the perturbations are large, this point is irrelevant for gradient calculations since only analytic grid derivative information is needed. However, since we employ a numerical optimization algorithm with line searches along a descent direction, true regridding is also necessary. For these line search calculations the grid perturbation algorithm is used so long as negative cell volumes are not created. If $\sin$ gularities begin to develop in the grid, the original grid generator can be used to create a new grid and the process restarted. In this work a modified version of WBGRID is used for automatic generation of the base grid, and subsequent reinitializations of the grid if needed.

After substitution of (26), the resulting expression for $\delta I$ is reduced to surface integrals in which the remaining unknowns are the grid metrics. These surface grid metrics can be easily determined for any modification in the surface by direct evaluation. This suggests choosing a set of design variables which smoothly modifies the original shape, say $b_{i}$. The gradient can then be defined with respect to these design variables as

$$
\mathcal{G}\left(b_{i}\right)=\frac{\delta I}{\delta b_{i}}
$$

where $\delta l$ is calculated by (25) with each term $b_{i}$ being independently perturbed by a finite step.

Therefore, to construct $\mathcal{G}$, a basis space of independent perturbation functions $b_{i}, \imath=1,2, \ldots, n$ ( $n=$ number of design variables) should be chosen that allows for the needed freedom of the design space. In this work design variables have been chosen with the following chordwise form, suggested by Hicks and Henne [11, 12]:

$$
b(x)=\sin \left(\pi x^{\frac{\log _{10}(3)}{\left.\log _{10}(t)\right)}}\right)^{t_{2}}
$$




$$
b(\bar{x})=\bar{x}^{t_{1}}(1-\bar{x}) e^{-t_{3} \bar{x}}
$$

where $t_{1}$ and $t_{2}$ control the center and width of the perturbation and $\bar{x}$ is the normalized chord length. When distributed over the entire chord on both upper and lower surfaces these analytic perturbation functions admit a large design space for each wing defining station. Then, by choosing a number of defining stations which reflects a practical design and linearly lofting the spanwise variations, the entire wing may be designed. An additional twist design variable is also included at each station to allow for washout that may be needed. These design variables have the advantage of being space-based functions, as opposed to frequency-based functions, and thus they allow for local control of the design. They can be chosen such that symmetry, thickness, or volume can be implicitly constrained. Further, particular choices of these variables will concentrate the design effort in regions where refinement is needed, while leaving the rest of the wing virtually undisturbed. The disadvantage of these functions is that they do not necessarily form a complete basis space when their number is increased, nor are they orthogonal. Thus, they do not guarantee that a solution, for example, of the inverse problem for a realizable target pressure distribution will necessarily be attained. Here, they are employed for their ease of use and ability to produce a wide variation of shapes with a limited number of design variables. Note that this limited set of design variables does not allow for planform modifications. However, here the interest is focused on Aerodynamic optimization and not on true Multi-Disciplinary optimization. In order to accomplish realistic planform design a true multi-point multi-disciplinary design method incorporating all aspects of aircraft direct operating cost would have to be used, because of trade-offs such as that between wing span and weight. Here the problem focuses on squeezing the most performance from a given planform.

\section{Implementation of the Euler based design methods}

Both of the design methods have been successfully implemented. The two techniques share many common features such as the flow and adjoint solution algorithms. The procedures can be summarized as follows.

1. Solve the flow equations (7-12) for $\rho, u_{i}, p, E$ $H$, and $I_{i}$

2. Smooth the cost function if necessary by (23).

3. Solve the adjoint equations (18) for $\psi$ subject to the boundary conditions (19).
4. Either calculate $\mathcal{P}, \mathcal{Q}$ and $\mathcal{R}$, by (21), from the variation in the control $S(\xi, \zeta)$, or evaluate the surface independent terms in equation (26).

5. Evaluate $\mathcal{G}$ by equation (22) or (27)

6. Then either correct the mapping $S(\xi, \zeta)$ or update the design variables $b_{i}$ based on the direction from steepest descent

$$
\delta S(\xi, \zeta)=-\lambda \mathcal{G} \text { or } \delta b_{i}=-\lambda \mathcal{G}
$$

or as an alternative a quasi-Newton method.

\section{Return to 1 .}

In practice these methods resemble those used by Hicks, Reuther et al. $[35,13,33]$ with control theory replacing the brute-force, finite difference gradient calculation. Unlike the earlier procedures, the current methods' computational costs do not hinge upon the number of design variables, which in these cases is either the number of surface mesh points used to represent $S(\xi, \zeta)$, or the number of perturbation functions $b_{i}$. Thus, with the three-dimensional implementation in hand, nonlinear aerodynamic design of complete aircraft can be brought into the realm of computational feasibility. The method also has the advantage of being quite general in that arbitrary choices for both the design variables and optimization technique are admitted.

The practical implementation of the design inethods rely heavily upon fast and accurate solvers for both the state $(w)$ and co-state $(\psi)$ fields. Further, to improve the speed and realizability of the methods, a robust choice of the optimization algorithm must be made. In the present case the second author's FLO87 computer program has been used as the basis of the design method. FLO87 solves the three-dimensional Euler equations with a cell-centered finite volume scheme, and uses residual averaging and multigrid acceleration to obtain very rapid steady state solutions, usually in 25 to 50 multigrid cycles $[16,17]$. Upwind biasing is used to produce nonoscillatory solutions, and assure the clean capture of shock waves. This is introduced through the addition of carefully controlled numerical diffusion terms, with a magnitude of order $\Delta x^{3}$ in smooth parts of the flow. The adjoint equations are treated in the same way as the flow equations. The fluxes are first estimated by central differences, and then modified by downwind biasing through numerical diffusive terms. These are supplied by the same subroutines as are used for the flow equations.

In the implementation with analytic mapping, a gradient procedure is used as the optimization process. However, to preserve the smoothness of the profile the gradient is smoothed at each step. Thus the change in the shape function $S(\xi, \zeta)$ is defined 
by solving

$$
\delta S-\frac{\partial}{\partial \xi} \beta \frac{\partial}{\partial \xi} \delta S=-\Lambda \mathcal{G}
$$

where $\beta$ is a smoothing parameter. Then, to first order, the variation in the cost is

$$
\begin{aligned}
\delta I & =\int \mathcal{G} \delta S d \xi \\
& =-\int \frac{1}{\Lambda}\left[\delta S-\delta S \frac{\partial}{\partial \xi} \beta \frac{\partial}{\partial \xi} \delta S\right] d \xi \\
& =-\int \frac{1}{\Lambda}\left[\delta S^{2}+\beta\left(\frac{\partial}{\partial \xi} \delta S\right)^{2}\right] d \xi \\
& <0 .
\end{aligned}
$$

Thus an improvement is still asured when smoothing is used. For the implementation on arbitrary meshes a quasi-Newton optimization method is employed. For this purpose the QNMDIF program developed by Gill, Murray and Wright [10] is used.

The option to minimize the pressure drag coefficient is realized in both methods by redefining the cost function as

$$
\begin{aligned}
I=C_{D}= & \frac{1}{\frac{1}{2} \rho_{\infty} q_{\infty}^{2} \mathbf{S}_{\text {ref }}} \iint_{B_{W}} p G_{21} d \xi_{1} d \xi_{3} \\
& +\frac{1}{\frac{1}{2} \rho_{\infty} q_{\infty}^{2} \mathbf{S}_{\text {ref }}} \iint_{B_{S}} p G_{31} d \xi_{1} d \xi_{2}
\end{aligned}
$$

where $\mathbf{S}_{\text {ref }}$ is the reference area. To prevent the procedure from trying to reduce drag by reducing the profile to a non-lifting flat plate a target pressure distribution can be retained in the cost function, which becomes

$$
I=\frac{1}{2} \Omega_{1} \iint_{B_{W}}\left(p-p_{d}\right) \delta p d \xi_{1} d \xi_{3}+\Omega_{2} C_{d}
$$

where $\Omega_{1}$ and $\Omega_{2}$ are weighting parameters. Similar constructions are employed for other cost functions such as $\left(1 / \frac{L}{D}\right)$.

\section{Numerical tests of the Three-Dimensional Method Using an Analytic Mapping}

The analytic grid generation method has been tested for the optimization of a swept wing [22, 21]. Two examples are presented here. In each the planform was fixed while the wing sections were free to be changed arbitrarily by the design method

In the first example the wing has a unit-semispan, with 36 degrees leading edge sweep. It has a compound trapezoidal planform, with straight taper from a root chord of 0.38 to a chord of 0.26 at the 30 percent span station, and straight taper from there to a chord of 0.12 at the tip, with an aspect ratio of 8.7. The initial wing sections were based on the Korn airfoil, which was designed for shock free flow at Mach 0.75 with a lift coefficient of 0.63 , and has a thickness to chord ratio of 11.5 percent [1]. The thickness to chord ratio was increased by a factor of 1.2 at the root and decreased by a ratio of 0.8 at the tip, with a linear variation across the span. The inboard sections were rotated upwards to give 3.5 degrees twist across the span.

The two-dimensional pressure distribution of the Korn airfoil at its design point was introduced as a target pressure distribution uniformly across the span. This target is presumably not realizable since it would imply a lifting wing with zero vortex drag, but it serves to favor the establishment of a relatively benign pressure distribution. The total inviscid drag coefficient, due to the combination of vortex and shock wave drag, was also included in the cost function. Calculations were performed with the lift coefficient forced to approach a fixed value by adjusting the angle of attack every fifth iteration of the flow solution. A grid with $192 \times 32 \times 48=294,912$ points was used, and the wing shape was determined by 133 sections each with 128 mesh points for a total of 4,224 design variables. It was found that the computational costs can be reduced by using only 15 multigrid cycles in each flow solution, and in each adjoint solution. Although this is not enough for full convergence, it proves sufficient to provide a shape modification which leads to an improvement. Figures 2, 3, and 4 shows the result of a calculation at Mach number of 0.82 , with the lift coefficient forced to approach a value of 0.5 . The plots show the initial wing geometry and pressure distribution, and the modified geometry and pressure distribution after 8 design cycles. The total inviscid drag was reduced from 0.0185 to 0.0118 . The initial design exhibits a very strong shock wave in the inboard region. It can be seen that this is completely eliminated, leaving a very weak shock wave in the outboard region.

To verify the solution, the final geometry, after 8 design cycles, was analyzed with another method, using the computer program FLO67. This program uses a cell-vertex formulation, and has recently been modified to incorporate a local extremum diminishing (LED) algorithm with a very low level of numerical diffusion [20]. When run to full convergence it was found that the initial wing has a drag coefficient of 0.0171 at $\mathrm{Mach} 0.82$ and a lift coefficient of 0.496 , with a corresponding lift to drag ratio of 29 . The result is illustrated in Figure 5. The final wing. shown in Figure 6, has a drag coefficient of 0.0107 with a lift coefficient of 0.497 , giving a lift to drag ratio of 47 at the same Mach number. A calculation at Mach 0.50 shows a drag coefficient of 0.0100 for a lift coefficient of 0.500 . Since in this case the flow is entirely subsonic, this provides an estimate of the vortex drag for this planform and lift distribution. 
Thus the design method has reduced the shock wave drag coefficient to about 0.0007 .

The second example is a design at a higher Mach number of 0.85 , with a target lift coefficient of 0.55 . This is a more severe test of the method, and a higher sweep back angle of 38 degrees at the leading edge was used. The wing has a modified trapezoidal planform, with straight taper from a root chord of 0.38 , and a curved trailing edge in the inboard region blending into straight taper outboard of the 30 percent span station to a tip chord of 0.10 , with an aspect ration of 9.0 . The initial wing sections were based on a section specially designed by the second author's two dimensional design method [18] to give shock free flow at Mach 0.78 with a lift coefficient of 0.6 . This section, which has a thickness to chord ratio of 9.5 percent, was used at the tip. Similar sections with an increased thickness were used inboard. The variation of thickness was nonlinear with a more rapid increase near the root, where the thickness to chord ratio of the basic section was multiplied by a factor of 1.47 . The inboard sections were rotated upwards to give the initial wing 3 degrees of twist from root to tip. The two dimensional pressure distribution of the basic wing section at its design point again was introduced as a target pressure distribution uniformly across the span. Figures 7 and 8 show the result of the optimization. The total inviscid drag coefficient was reduced from 0.0243 to 0.0144 . The results of this optimization were also verified by calculations with FLO67, using a high resolution LED algorithm. Figures 9 and 10 show that when the solutions were fully converged the drag coefficient was reduced from 0.0236 to 0.0119 , with an improvement in lift to drag ratio from 23 to 46. The result is illustrated in figure 10. A subsonic calculation at Mach 0.50 shows a drag coefficient of 0.0109 for the same lift coefficient of 0.55 . Thus in this case the shock wave drag coefficient is about 0.0010 .

\section{Numerical tests of the Three-Dimensional Method for Wing and Wing-Body Configurations Using a General Mesh}

The first design case involving the arbitrary mesh implementation is an inverse design to obtain the ONERA-M6 wing at $M=0.84$ and $\alpha=3.06^{\circ}$. The fixed alpha design starts from the ONERA-M6 planform but has NACA 0012 airfoil sections instead of the original sections. The goal is thus to recover the original ONERA-M6 sections by prescribing its actual pressure distribution at the desired conditions as the target. Design variables are specified at 6 defining stations which are then lofted in the span- wise direction. Twenty-five design variables are used at each defining station, for a total of 150 . They are specified such that only thickness as a function of $\bar{x}$ can be adjusted. This choice reflects the fact that both the initial and final designs are characterized by symmetrical sections. The pressure distributions and airfoil sections for both the initial condition and the target are shown in Figure 11. Figure 12 displays the solution after 19 design iterations. The target pressure distribution is almost obtained over most of the wing, with small discrepancies occurring close to the leading edge. There are no discernible differences between the final airfoils and their targets. It is possible that there are not enough basis functions to allow exact recovery of the target pressure. However, the discrepancies may also result simply from the fact that it would take a considerably greater number of design iterations to obtain better convergence to a more precise minimum. The design process was stopped when the rate of reduction in the cost function between design iterations slowed considerably.

To explore the wing-body design capability of the general mesh formulation, a DC-9-30 planform is used as a testbed. The DC-9-30 is characterized by a straight tapered wing with an aspect ratio of 8.7, $\frac{1}{4}$ chord sweep of $24.5^{\circ}$, washout of $4.5^{\circ}$ and a taper ratio of 0.203 . The fuselage has a diameter of $11.2 \mathrm{ft}$ and a length of $107.6 \mathrm{ft}$. Figure 13 shows the surface mesh generated by WBGRID for the DC:-9. Although the DC-9 cruises at a variety of different altitudes and Mach numbers its high speed cruise is $M=0.78$.

In our first attempt to redesign the wing contours for the DC-9-30, our starting point uses NAC A $64 \mathrm{~A} 410$ airfoil defined at 9 stations across the span and scaled to $12.6 \%$ thick at the root and $9.4 \%$ thick at the tip. Even though we are using a relatively fine $161 \times 41 \times 41$ mesh, a glance at Figure 13 shows that the body mesh is still relatively coarse. But since body drag should stay essentially negligible for inviscid calculations, the inverse of wing $L / D$ was used as the cost function. This amounts to wing optimization in the presence of the body. Both airfoil sections and pressure distributions for the initial condition are shown in Figure 14. The NACA $64 \mathrm{~A} 410$ airfoil sections cause a strong shock across the entire upper surface of the wing since it was never intended for transonic use. A total of 117 design variables are used to modify the wing. Nine design variables adjust the twist at each wing defining station, while 54 design variables modify the leading edge shape and another 54 design variables alter the camber. The use of such a mix of variables demonstrates the flexibility of our choice of basis functions. The current choice fixes the planform and maximum thickness distribution but allows for a large range of wing shapes. Further, since the majority of the wing 
is designed via variables that alter camber and twist, no prescribed pressure distributions were necessary to retain thickness.

The wing was optimized in the constant $C_{L}$ mode and after 16 design iterations it is shown in Figure 15. The pressure distributions demonstrate that a significant reduction in the shock strength has been realized at the same time that the leading edge of the wing has been properly loaded. The wing drag coefficient has been reduced from 0.0248 to 0.0155 while the wing $L / D$ has increased from 18.28 to 28.87 . As in the case for the analytic mesh wing designs, a subsonic case was analyzed to determine the level of induced drag present in the configuration. These calculations revealed that the configuration contains 149 counts of induced drag on the wing; thus the approximate wave drag coefficient for the optimized design is 0.0006

This calculation used 4.8 hours of Cray C-90 single processor time including all flow and adjoint calculations. An estimate of the comparable calculation using finite differences for the gradient calculations is 160 hours. The calculation therefore shows a factor of 33 savings in C.PU time through the use of continuous sensitivities.

A second attempt to redesign the DC-9 wing at a Mach number of 0.80 represents a more difficult challenge since it pushes the $24.5^{\circ}$ swept wing well past its design conditions. Again 117 design varjables were specified such that twist, leading edge shape, and camber could be modified. The initial NACA $64 A 410$ airfoil sections and pressure distributions are shown in Figure 16. Figure 17 shows the result after 15 design iterations where $C_{D_{w}}$ has been reduced from 0.0315 to 0.0167 and the wing lift to drag ratio has been increased from 14.44 to 26.86 . The remaining component of the coefficient of drag due to shock waves for this design is about 0.0018 . While this shows a dramatic performance improvement over the original wing it is still not quite as good as the wing designed at $M=0.78$. This indicates that it may be difficult to obtain a very good design at this higher Mach number without allowing a change in thickness, a change in sweep, or a reduction in the operating lift coefficient. An examination of the pressures reveals that this design is probably incurring the additional drag because of a stronger shock wave that traverses the span.

\section{Conclusions and Recommendations}

In the period since this approach to optimal shape design was first proposed by the author [18], the method has been verified by numerical implementation for both potential flow and flows modeled by the Euler equations $[19,34,23]$. It has been demonstrated that it can be successfully used with a finite volume formulation to perform calculations with ar- bitrary numerically generated grids $[34,23]$. Here results are presented for three-dimensional calculations using both the analytic mapping and general finite volume implementations. The design of both wing and wing-body configurations indicates that this approach has matured to the extent that it can be a useful tool for the design of new aircraft. A factor of 33 savings in CPU time has been accomplished through the use of the adjoint formulation The clear limitation in these current results is the reliance on a single structured block for both the state and co-state fields. In the future our group intends not only to extend the method to treat both multiblock and unstructured meshes, but also to implement a Navier-Stokes version.

\section{ACKNOWLEDGMENTS}

This research has benefited greatly from the generous support of the AFOSR under grant number AFOSR-91-0391, ARPA under grant number N00014-92-J-1976, USRA through RIACS, the High Speed Research branch of NASA Ames Research Center and IBM. Considerable thanks also goes to David Saunders of Sterling Software for his assistance in assembling the text.

\section{References}

[1] F. Bauer, P. Garabedian, D. Korn, and A. Jameson. Supercritical Wing Sections II. Springer Verlag, New York, 1975.

[2] O. Baysal and M. E. Eleshaky. Aerodynamic design optimization using sensitivity anaysis and computational fluid dynamics. $A I A A p a-$ per 91-0471, 29th Aerospace Sciences Meeting. Reno, Nevada, January 1991.

[3] O. Baysal and M. E. Eleshaky. Aerodynamic sensativity analysis methods for the compressible Euler equations. Journal of Fluids Engineering, 113(4):681-688, 1991.

[4] O. Baysal and M. E. Eleshaky. Aerodynamic design optimization using sensitivity anaysis and computational fluid dynamics. AIAA Journal, 30(3):718-725, 1992 .

[5] O. Baysal and M. E. Eleshaky. Airfoil shape optimization using sensitivity analysis on viscous flow equations. Journal of Fluids Engineering, $115: 75-84,1993$.

[6] (i. W. Burgreen and O. Baysal. Aerodynamic shape optimization using preconditioned conjugate gradient methods. AIA A paper 93-3920, July 1993. 
[7] Ci. W. Burgreen and O. Baysal. Threedimensional aerodynamic shape optimization of wings using sensitivity analysis. $A I A A$ paper 94-0094, 32nd Aerospace Sciences Meeting and Exhibit, Reno, Nevada, January 1994.

[8] M. E. Eleshaky and O. Baysal. Preconditioned domain decomposition scheme for threedimensional aerodynamic sensitivity analysis. In Proceedings of of the Ioth $A I A A$ computational fluid dynamics conference, pages 10551056, July 1993.

[9] M. E. Eleshaky and O. Baysal. Shape optimization of a 3 -d nacelle near a flat plate wing using multiblock sensitivity analysis. $A I A A \mathrm{pa}$ per 94-0160, 32nd Aerospace Sciences Meeting and Exhibit, Reno, Nevada, January 1994.

[10] P.E. Gill, W. Murray, and M.H. Wright. Practical Optimization. Academic Press, 1981.

[11] R. M. Hicks and P. A. Henne. Wing design by numerical optimization. Journal of Aircraft, 15:407-412, 1978 .

[12] R. M. Hicks and P. A. Henne. Wing design by numerical optimization. AlAA paper 79-0080, 1979 .

[13] R. M. Hicks, E. M. Murman, and G. N. Vanderplaats. An assessment of aifoil design by numerical optimization. NASA TM X-3092, Ames Research Center, Moffett Field, California, July 1974.

[14] W.P. Huffman, R.G, Melvin, D.P. Young, F.T. Johnson, J.E. Bussoletti, M.B. Bieterman, and C.L. Hilmes. Practical design and optimization in computational fluid dynamics. $A / A A p a-$ per 93-3111, AlAA 24th Fluid Dynamics ('onference, Orlando, Florida, July 1993.

[15] A. C. Taylor III, G. W. Hou, and V. M. Korivi. Sensitivity analysis, approximate anaysis, and design optimization for internal and external viscous flows. AlAA paper 91-3083, September 1991 .

[16] A. Jameson. Solution of the Euler equations for two dimensional transonic flow by a multigrid method. Applied Mathematics and Computalions, 13:327-356, 1983.

[17] A. Jameson. Multigrid algorithms for compressible flow calculations. In W. Hackbusch and U. Trottenberg, editors, Lecture Notes in Mathematics, Vol. 1228, pages 166-201. Proceedings of the 2nd European Conference on Multigrid Methods, Cologne, 1985, Springer-Verlag, 1986.
[18] A. Jameson. Aerodynamic design via control theory. Journal of Scientific Computing, 3:233$260,1988$.

[19] A. Jameson. Automatic design of transonic airfoils to reduce the shock induced pressure drag. In Proceedings of the 31 st Israel Annual Conference on Aviation and Aeronautics, Tel Aviv, pages 5-17, February 1990.

[20] A. Jameson. Artificial diffusion, upwind biasing, limiters and their effect on accuracy and multigrid convergence in transonic and hypersonic flows. AIAA paper 93-3359, AlAA 11 th Computational Fluid Dynamics Conference, Orlando, Florida, July 1993.

[21] A. Jameson. Aerodynamic design methods. In International Workshop on Solution Techniques for Large-Scale CFD Problems, Montreal, September 1994. CERC'A.

[22] A. Jameson. Optimum aerodynamic design via boundary control. In AGARD-VKI Lecture Series, Optimum Design Methods in Aerodynamics. von Karman Institute for Fluid Dynamics, 1994.

[23] A. Jameson and J. Reuther. Control theory based airfoil design using the Euler equations. $A I A A$ paper 94-4272, 5 th AIAA/USAF/NASA/ISSMO Symposium on Multidisciplinary Analysis and Optimization, Panama City Beach, FL, September 1994.

[24] V. M. Korivi, A. ( $($ Taylor III, (i. W. Hou, P. A. Newman, and H. E. Jones Sensitivity derivatives for three-dimensional supersonic Euler code using incremental iterative strategy. In Proceedings of of the 12th AIAA computational fiuid dynamics conference, pages 10531054, July 1993 .

[25] V. M. Korivi, A. C. Taylor III, and P. A. Newman. Aerodynamic optimization studies using a 3-d supersonic Euler code with efficient calculation of sensitivity derivatives. AIAA paper 94-4270, 5th AIAA/USAF/NASA/ISSMO Symposium on Multidisciplinary Analysis and Optimization, Panama City, Florida, September 1994 .

[26] V. M. Korivi, A. (: Taylor III, P. A. Newman, (i. W. Hou, and H. E. Jones. An incremental strategy for calculating consistent discrete CFD sensitivity derivatives. NASA TM 104207, Langley Research Center, Hampton, VA, February 1992.

[27] G. Kuruvila, S. Ta'Asan, and M. D. Salas. Airfoil optimization by the one-shot method. In 
AGARD-VKI Lecture Series, Optimum Design Methods in Aerodynamics. von Karman Institute for Fluid Dynamics, 1994.

[28] J.M. Lacasse and O. Baysal. Design optimization of single- and two-element airfoils on multiblock grids. AIAA paper 94-4273, 5th AIAA/USAF/NASA/ISSMO Symposium on Multidisciplinary Analysis and Optimization, Panama City, Florida, September 1994.

[29] J. L. Lions. Optimal Control of Systems Governed by Partial Differential Equations. Springer-Verlag, New York, 1971. Translated by S.K. Mitter.

[30] P. A. Newman, G. W. Hou, H. E. Jones, A. C. Taylor III, and V. M. Korivi. Observations on computational methodologies for use in large-scale gradient-based, multidisciplinary design incorporating advanced CFD codes. NASA TM 104206, Langley Research Center, Hampton, VA, February 1992.

[31] O. Pironneau. Optimal Shape Design for Elliptic Systems. Springer-Verlag, New York, 1984.

[32] O. Pironneau. Optimal shape design for aerodynamics. In AGARD-VKI Lecture Series, Optimum Design Methods in Aerodynamics. von Karman Institute for Fluid Dynamics, 1994.

[33] J. Reuther, S. Cliff, R. Hicks, and C.P. van Dam. Practical design optimization of wing/body configurations using the Euler equations. AIA A paper 92-2633. 1992.

[34] J. Reuther and A. Jameson. Control theory based airfoil design for potential flow and a finite volume discretization. AIAA paper 94$0499,32 \mathrm{nd}$ Aerospace Sciences Meeting and Exibit, Reno, Nevada, January 1994

[35] J. Reuther, C.P. van Dam, and R. Hicks. Subsonic and transonic low-Reynolds-number airfoils with reduced pitching moments. Journal of Aircraft, 29:297-298, 1992.

[36] S. Ta'asan, G. Kuruvila, and M. D. Salas. Aerodynamic design and optimization in one shot. AIAA paper 91-005, 30th Aerospace Sciences Meeting and Exibit, Reno, Nevada, January 1992.

[37] D.P. Young, W.P. Huffman, R.G. Melvin, M.B. Bieterman, C.L. Hilmes, and F.T. Johnson. Inexactness and global convergence in design optimization. AIAA paper 94-4286, 5 th AIAA/USAF/NASA/ISSMO Symposium on Multidisciplinary Analysis and Optimization, Panama City, Florida, September 1994. 

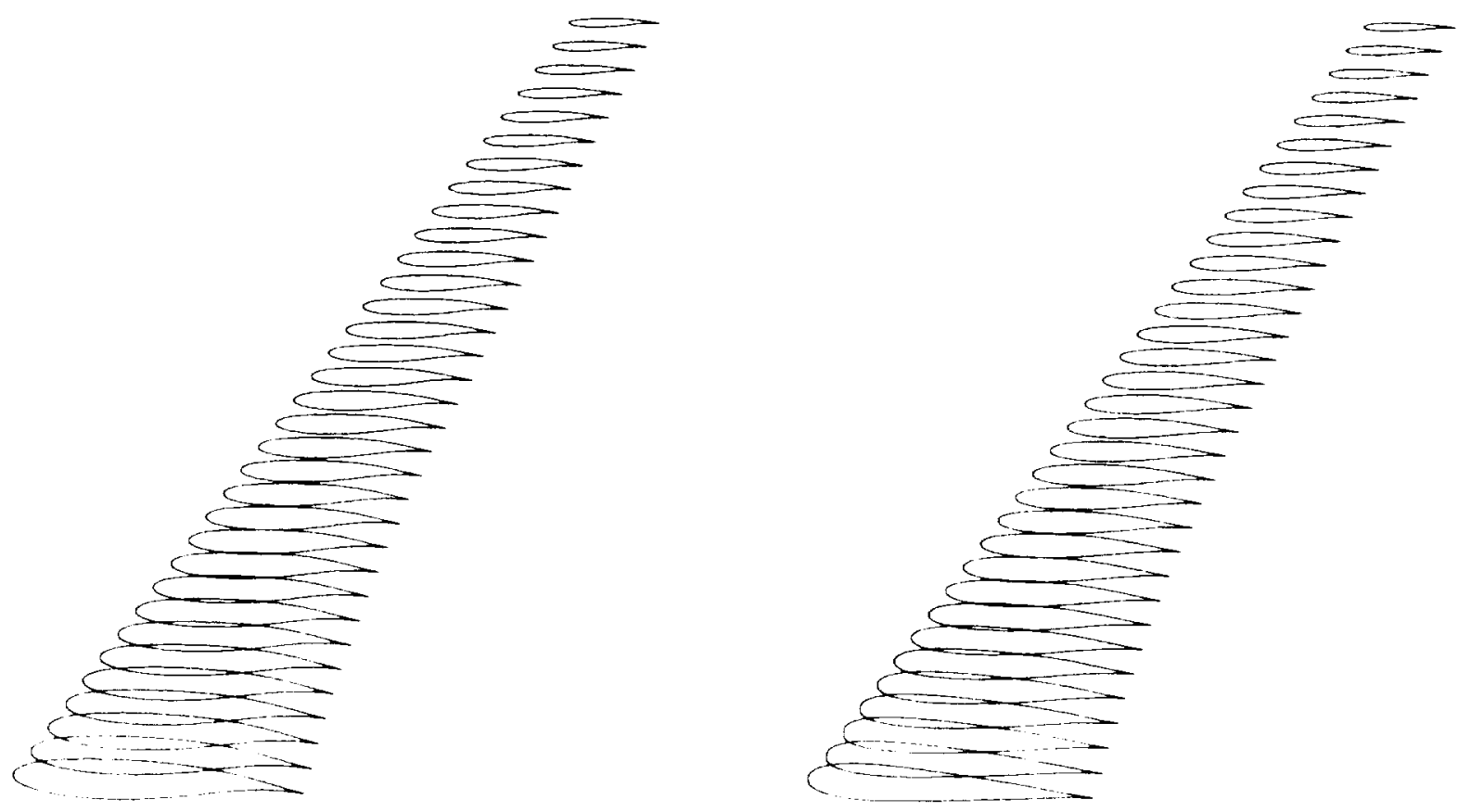

2a: Initial Wing

$C_{i}=0.5001, C_{d}=0.0185, \alpha=-0.958^{\circ}$

2b: 8 Design Iterations

$C_{l}=0.4929, C_{d}=0.0118, \alpha=0.172^{\circ}$

Figure 2: Lifting Design Case, $M=0.82$, Fixed Lift Mode.

Drag Reduction 


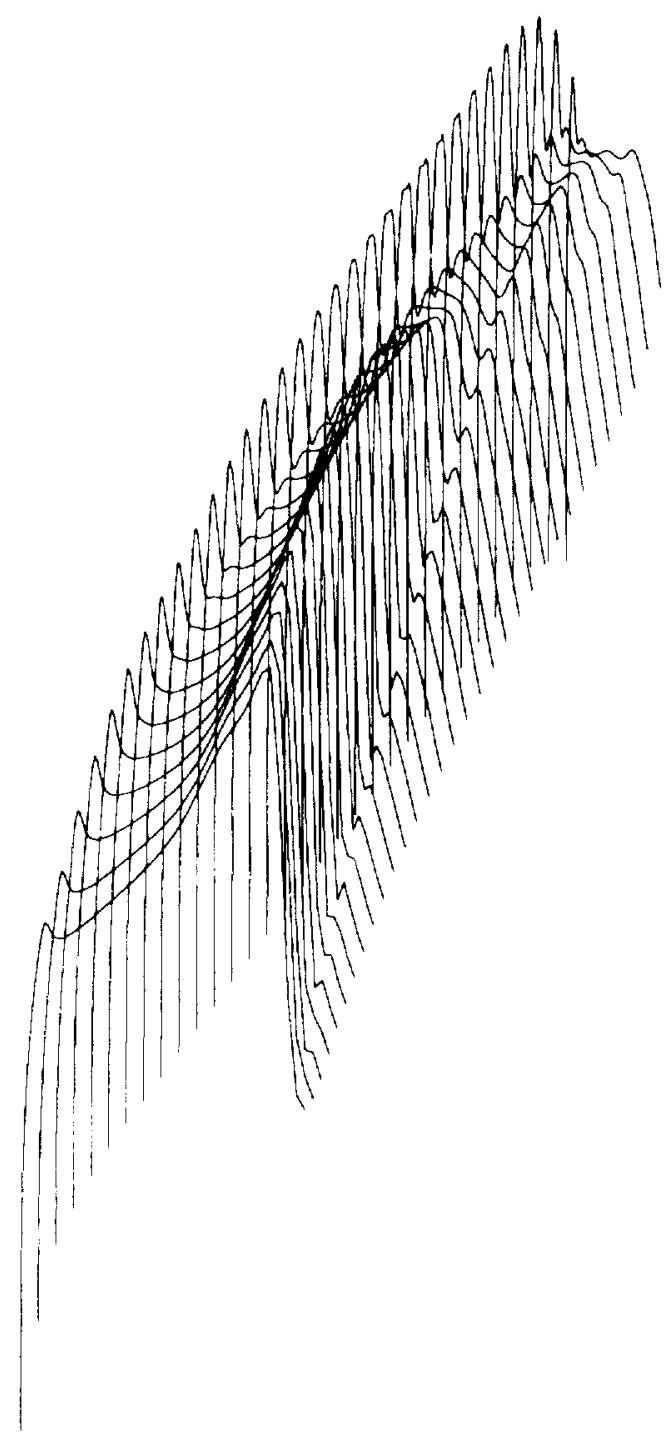

UPPER SURFACE PRESSURE

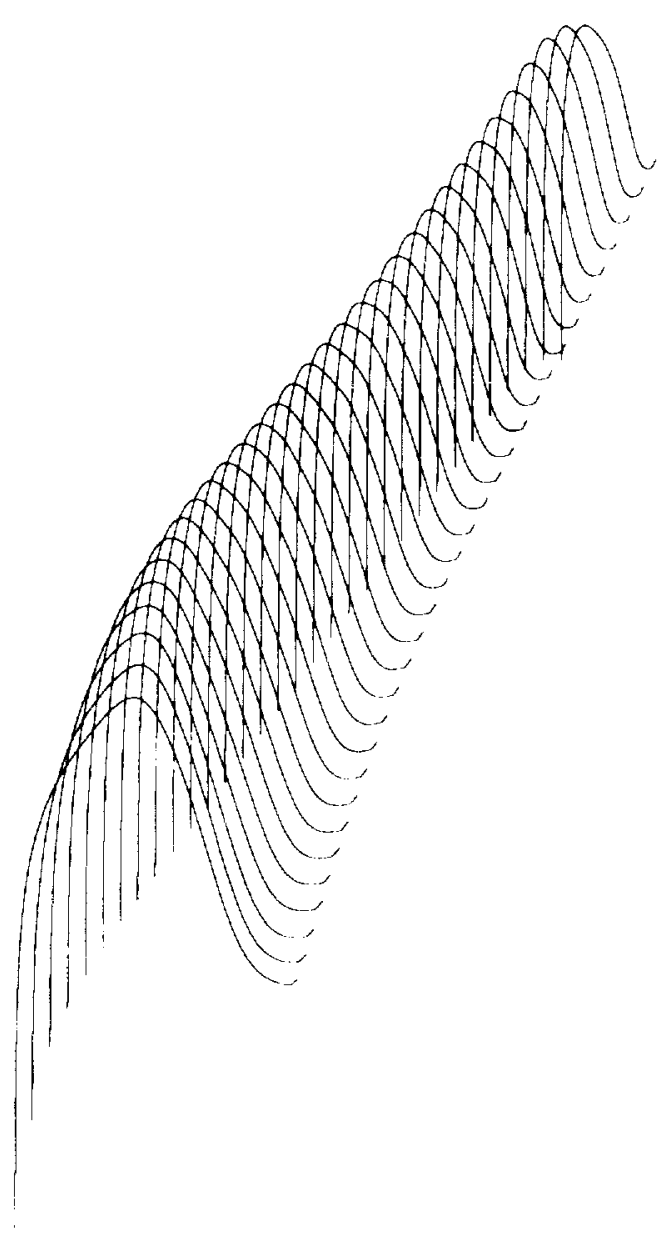

LOWER SURFACE PRESSURE

Figure 3: Lifting Design Case, $M=0.82$, Fixed Lift Mode. Initial Wing: Modfied Korn

$$
\begin{gathered}
C_{L}=0.5001, C_{D}=0.0185, \alpha=-0.958^{\circ} \\
\text { Drag Reduction }
\end{gathered}
$$




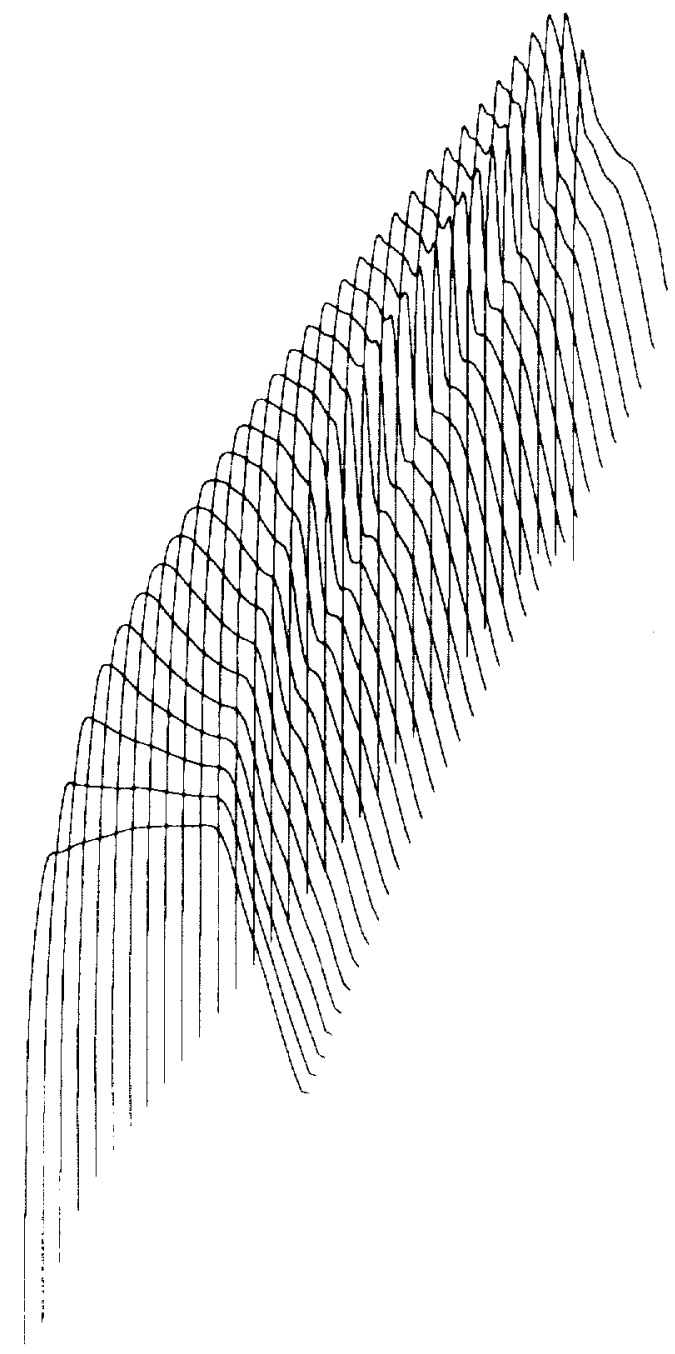

UPPER SURFACE PRESSURE

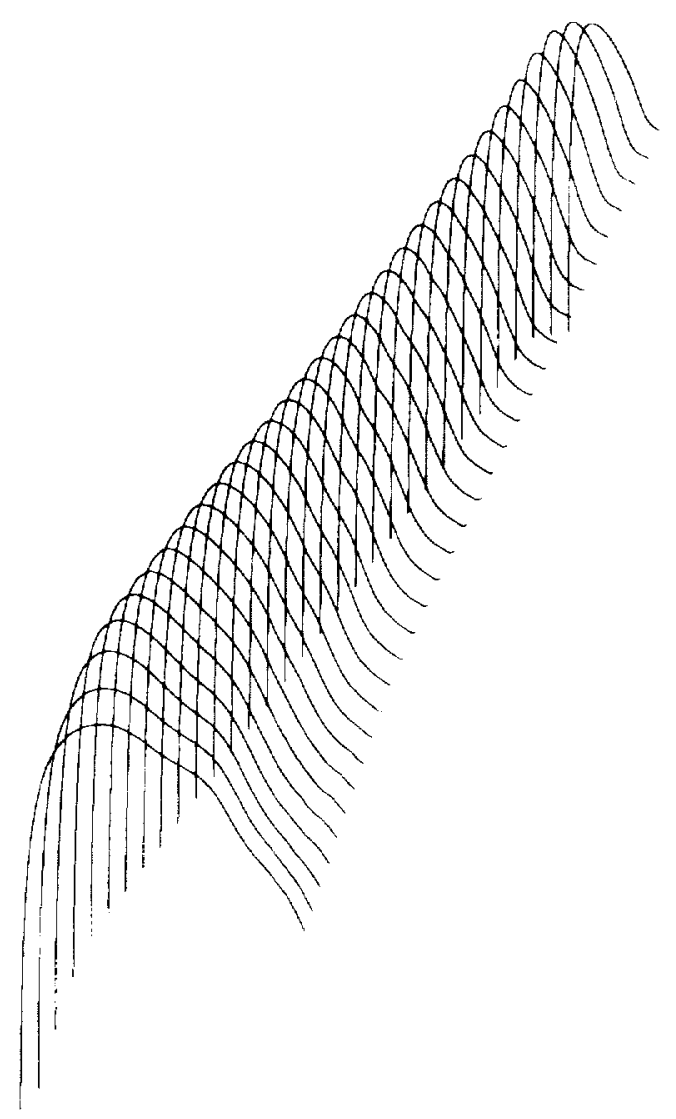

LOWER SURFACE PRESSURE

Figure 4: Lifting Design Case, $M=0.82$, Fixed Lift Mode.

$$
\begin{gathered}
\text { Design after } 8 \text { cycles } \\
C_{L}=0.4929, C_{D}=0.0118, \alpha=0.172^{\circ} \\
\text { Drag Reduction }
\end{gathered}
$$




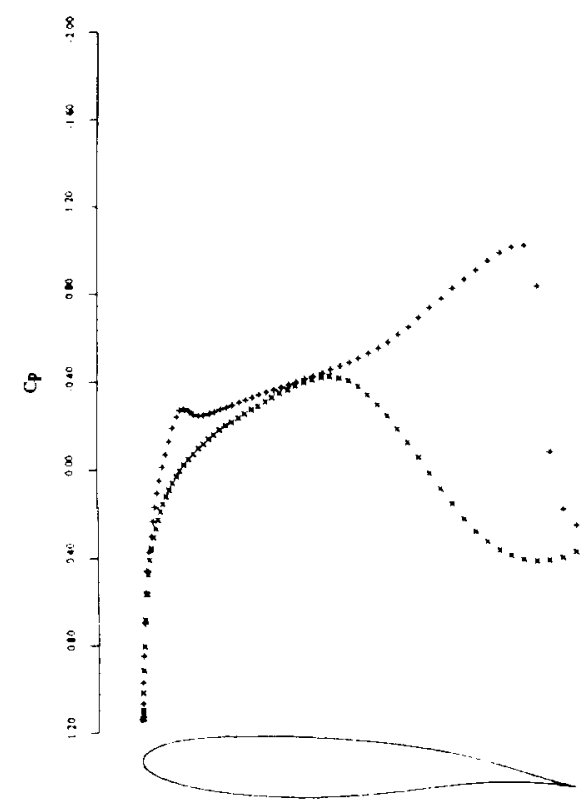

5a: span station $z=0.00$

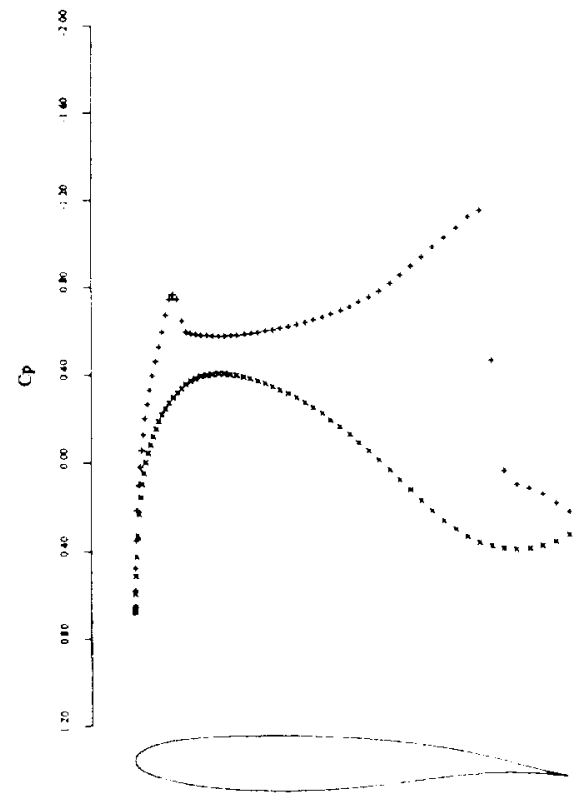

5b: span station $z=0.25$

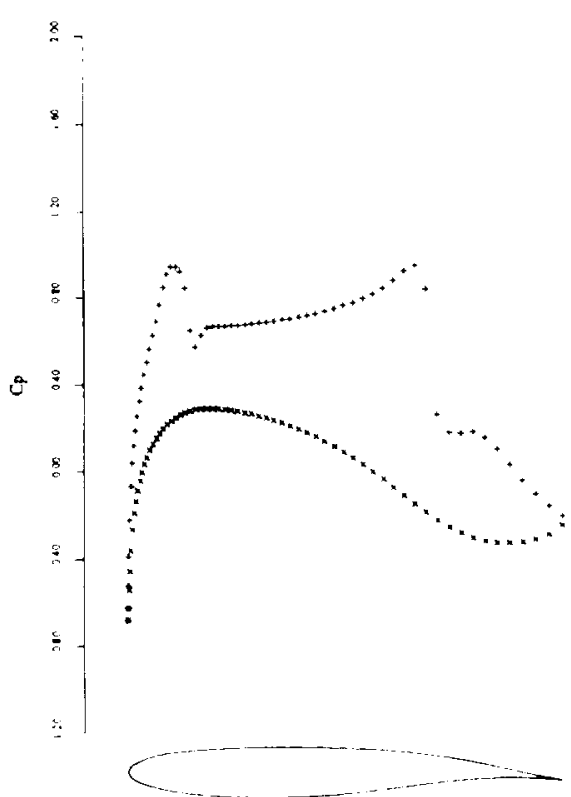

5c. span station $z=0.50$

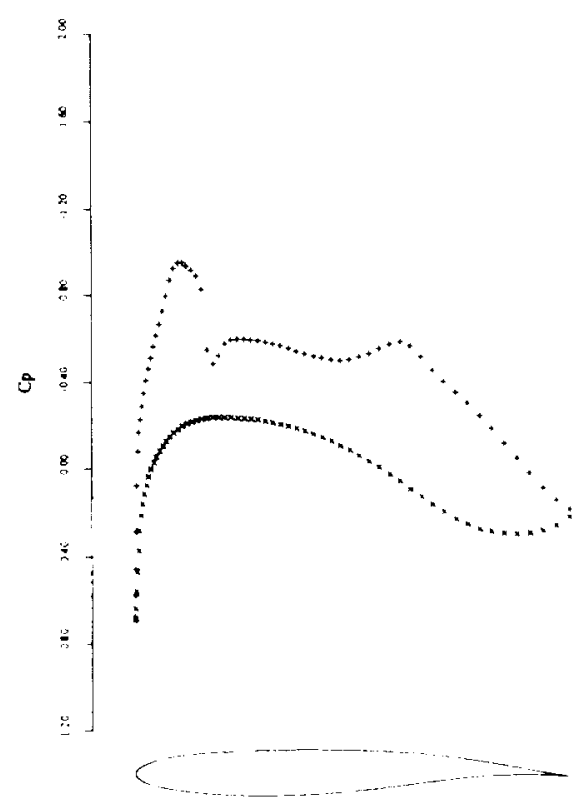

5 d. span station $z=0.75$

Figure 5: FLO67 check on initial wing

$M=0.82, C_{L}=0.4959, C_{D}=0.0171, \alpha=-1.080^{\circ}$ 


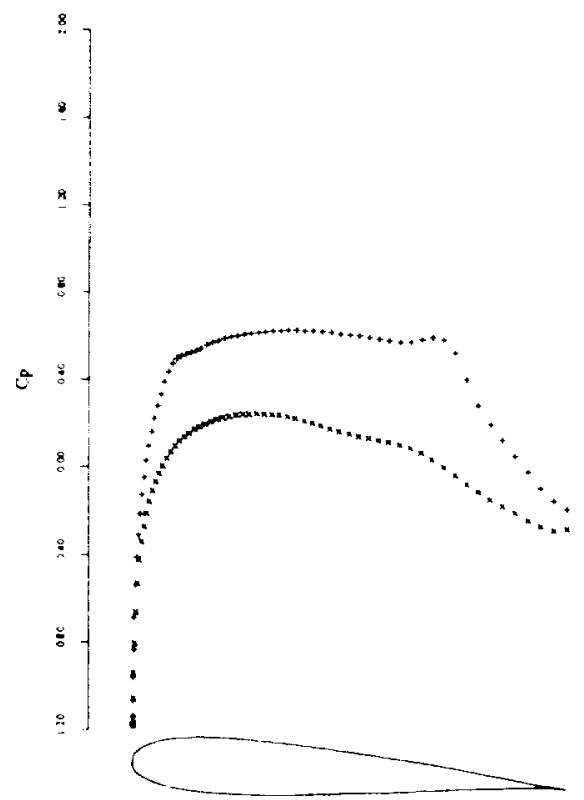

$6 a:$ span station $z=0.00$

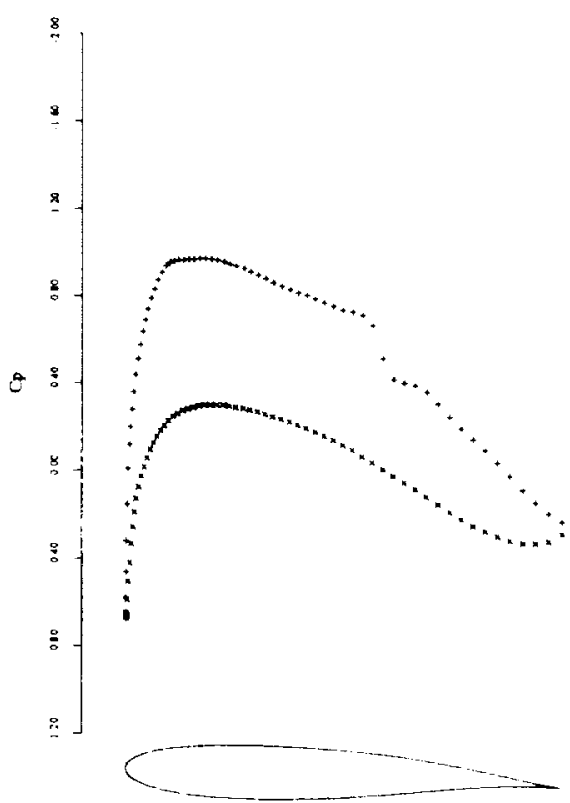

6b: span station $z=0.25$

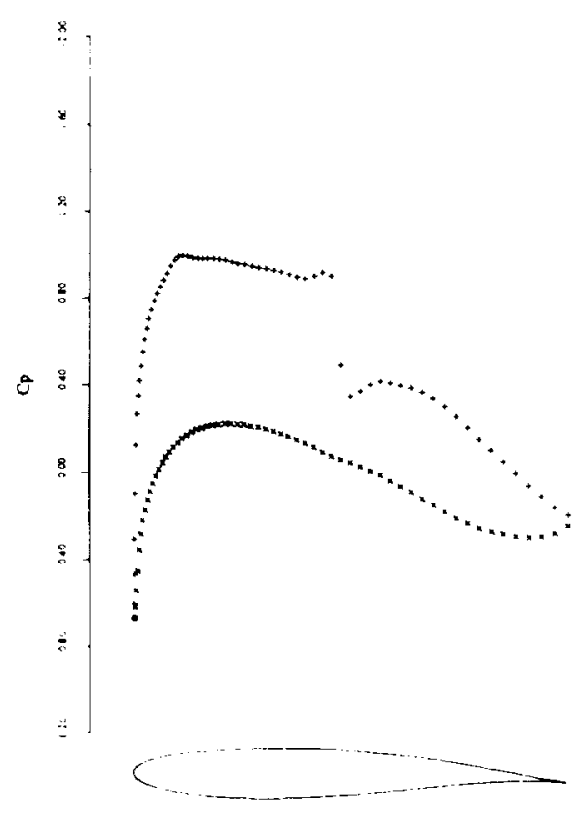

$6 c:$ span station $z=0.50$

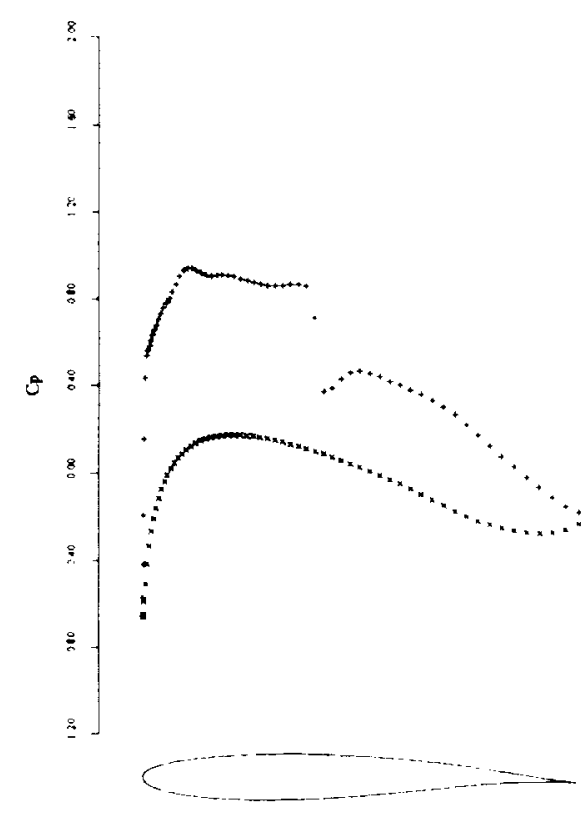

$6 \mathrm{~d}:$ span station $z=0.75$

Figure 6: FLO67 check on redesigned wing. $M=0.82, C_{L}=0.4975, C_{D}=0.0107, \alpha=0.200^{\circ}$ 

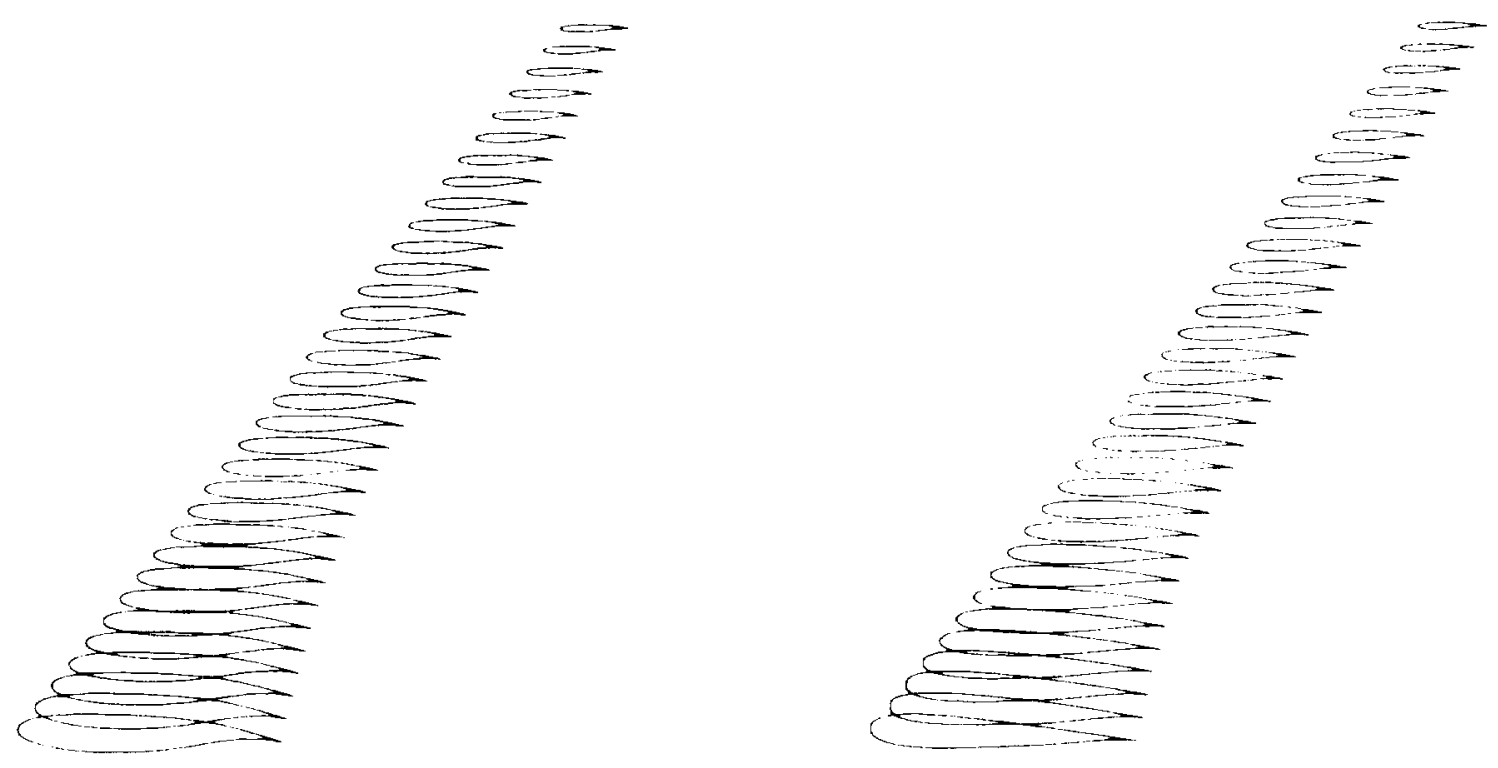

7a: Initial Wing

$C_{i}=0.5500, C_{d}=0.0243, \alpha=-0.962^{\circ}$

7b: 10 Design Iterations

$C_{i}=0.5500, C_{d}=0.0144, \alpha=0.274^{\circ}$

Figure 7: Lifting Design Case, $M=0.85$, Fixed Lift Mode.

Drag Reduction 


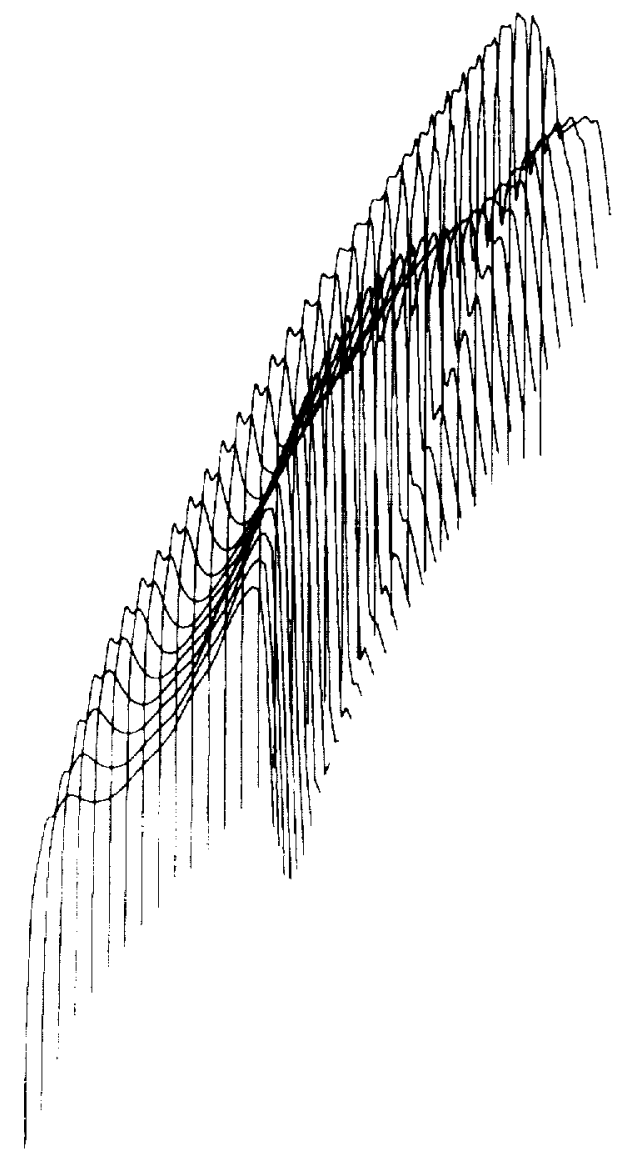

UPPER SURFACE PRESSURE

8a: Initial Wing

Lifting Design Case, $M=0.85$. Fixed Lift Mode

$C_{L}=0.5500, C_{D}=0.0243, \alpha=-0.962^{\circ}$

Drag Reduction

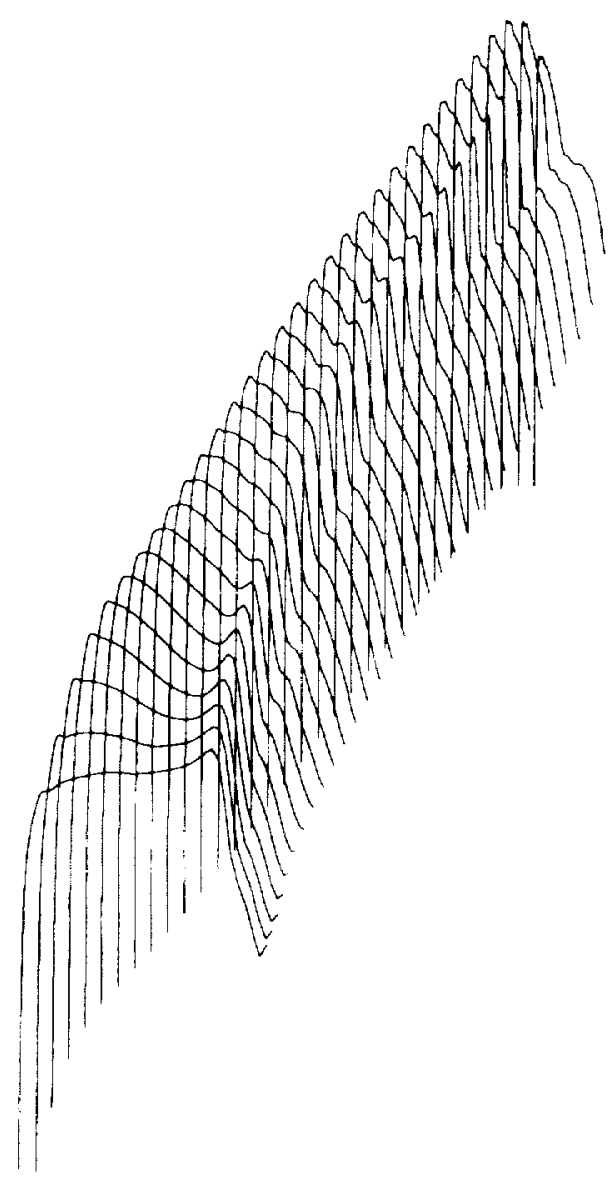

UPPER SURFACE PRESSURE

8b: 10 Design Iterations

Lifting Design Case, $M=0.85$, Fixed Lift Mode

$C_{L}=0.5500, C_{D}=0.0144, \alpha=0.274^{\circ}$

Drag Reduction

Figure 8: Lifting Design Case, $M=0.85$, Fixed Lift Mode.

Drag Reduction 


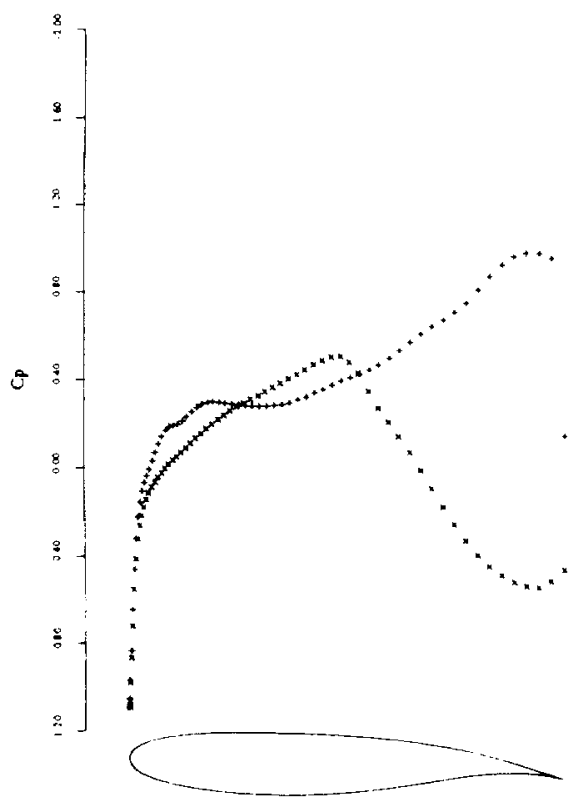

9a: span station $z=0.00$

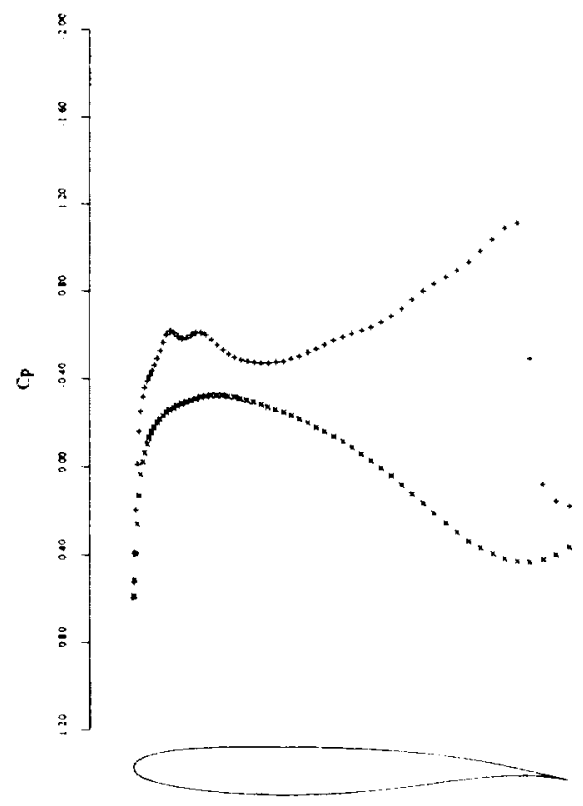

9b: span station $z=0.312$

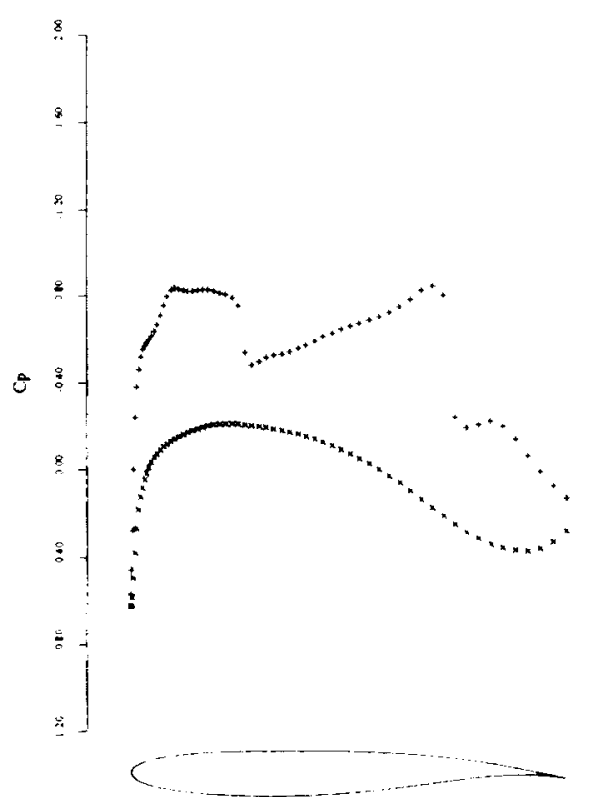

9c: span station $z=0.625$

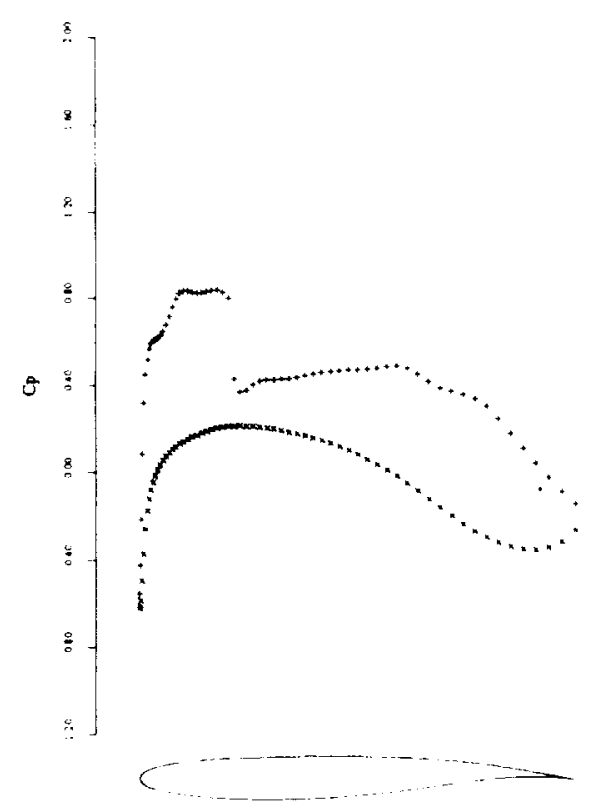

9d: span station $z=0.937$

Figure 9: FLO67 check on initial wing $M=0.85, C_{L}=0.5506, C_{D}=0.0236, \alpha=-1.260^{\circ}$. 


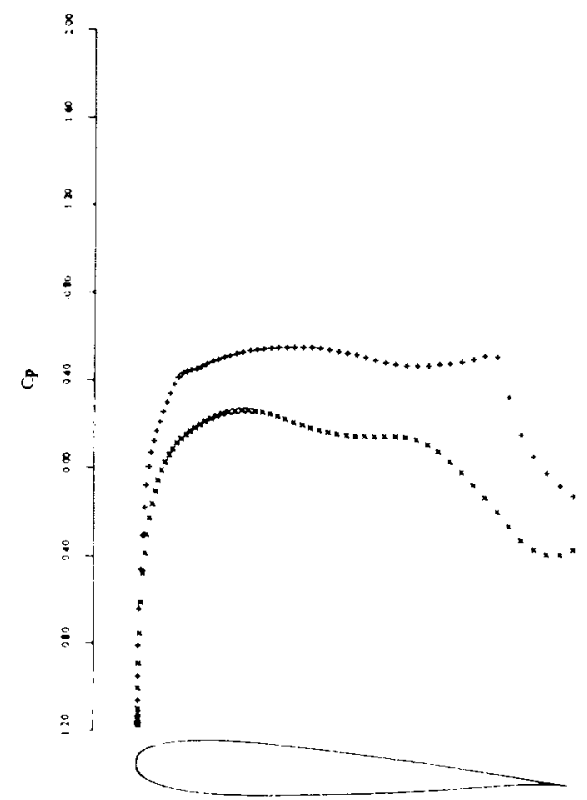

10a: span station $z=0.00$

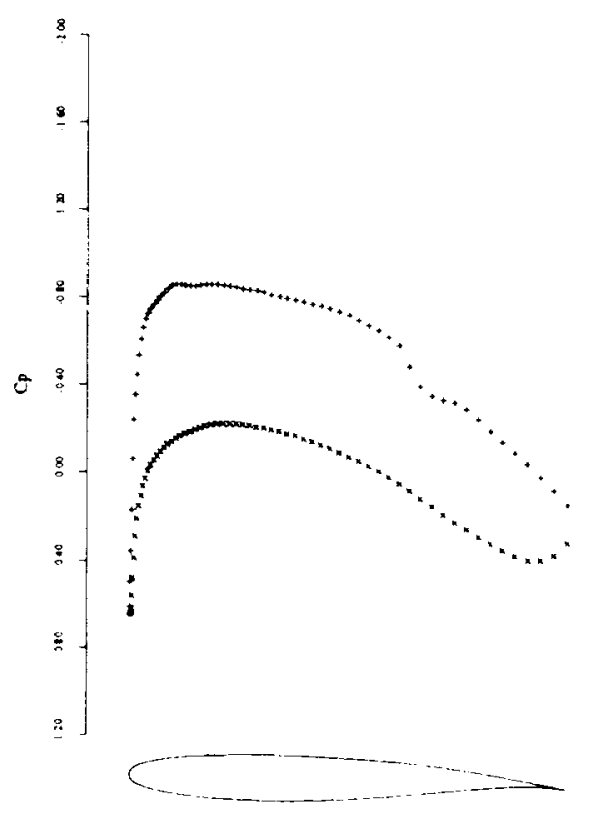

10b: span station $z=0.312$

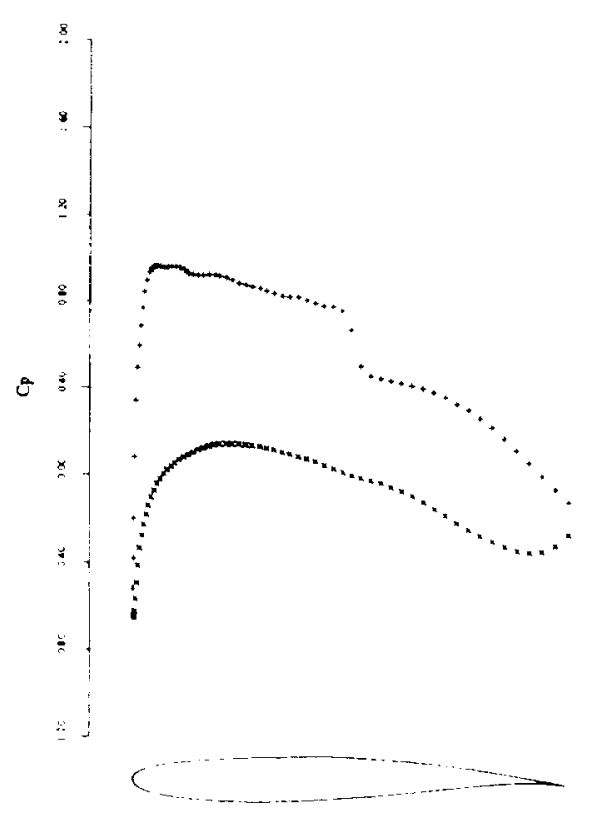

10c: span station $z=0.625$

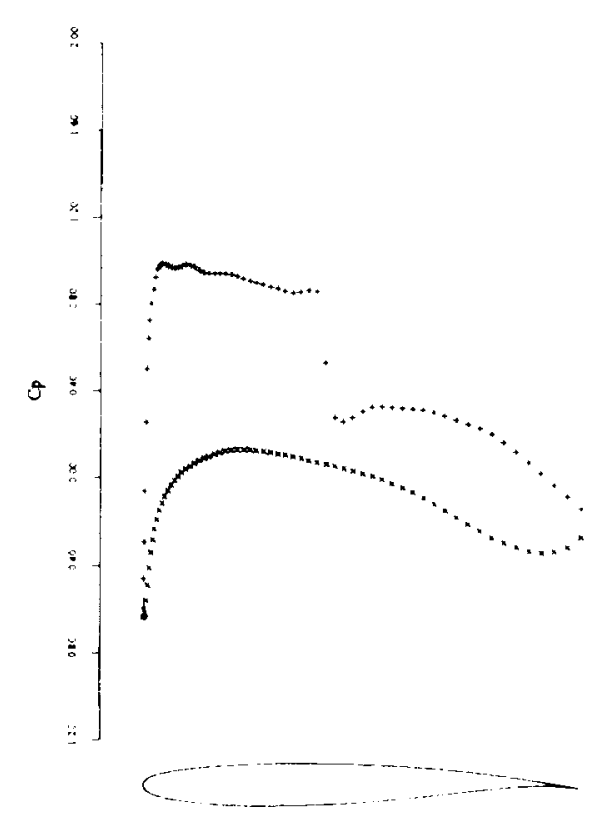

10d: span station $z=0.937$

Figure 10: FLO67 check on redesigned wing $M=0.85, C_{L}=0.5500, C_{D}=0.0119, \alpha=0.210^{\circ}$ 


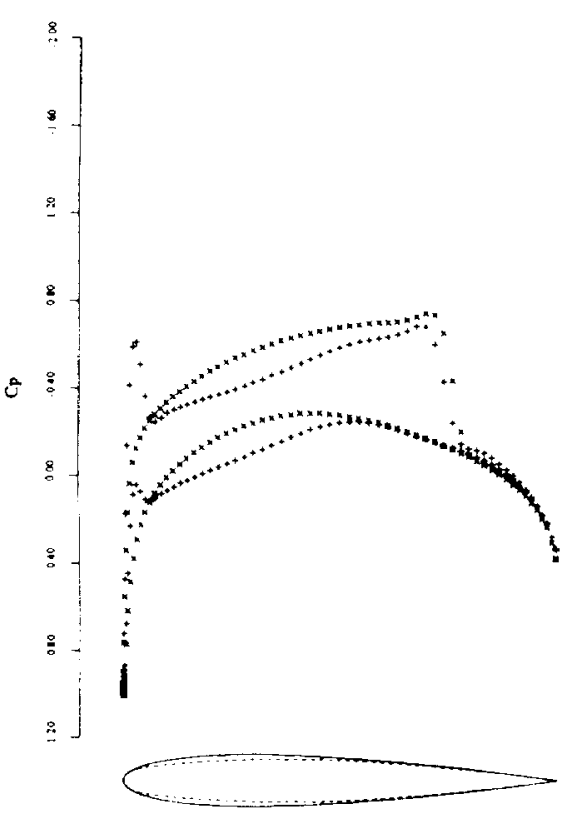

11 a: span station $z=0.021$

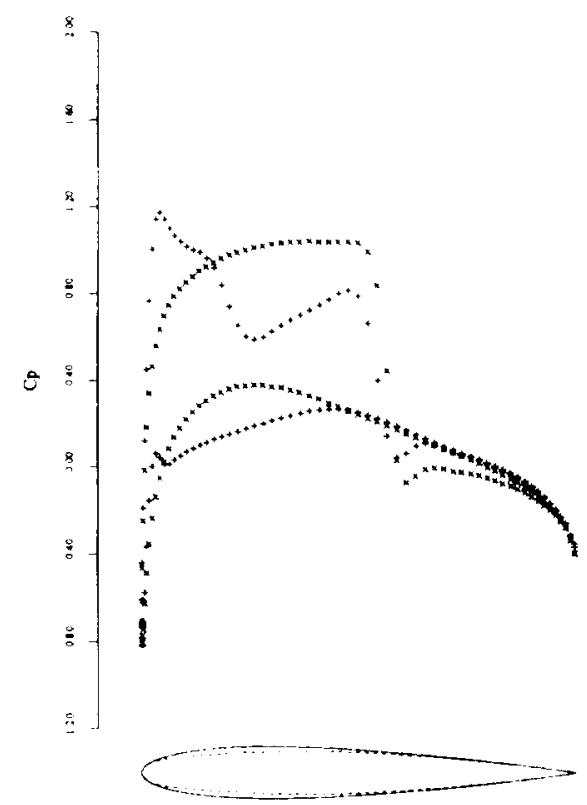

11c: span station $z=0.604$
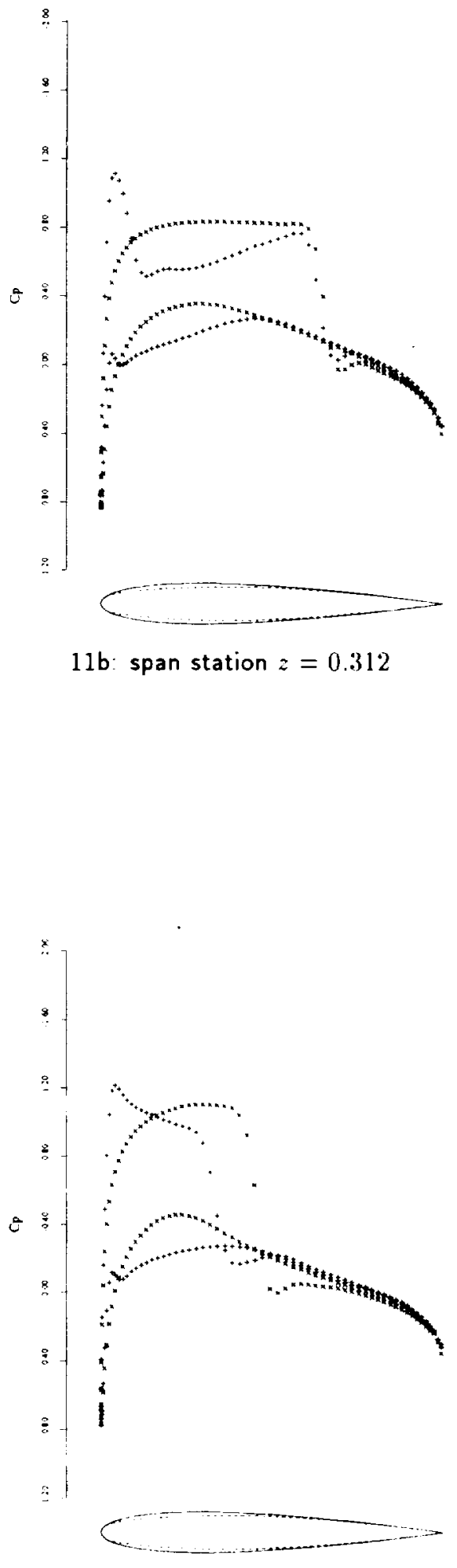

11d: span station $z=0.896$

Figure 11: Initial condition for ONERA-M6 design. $M=0.84, C_{L}=0.1723, C_{D}=0.0122, \alpha=3.060^{\circ}$

,$- \times$ Initial Wing: NACA 0012

- - . + Target $C_{p}$ : ONERA-M6.

150 Design Variables, $161 \times 41 \times 41$ Mesh, Euler Wing/Body Solution Inverse Design 


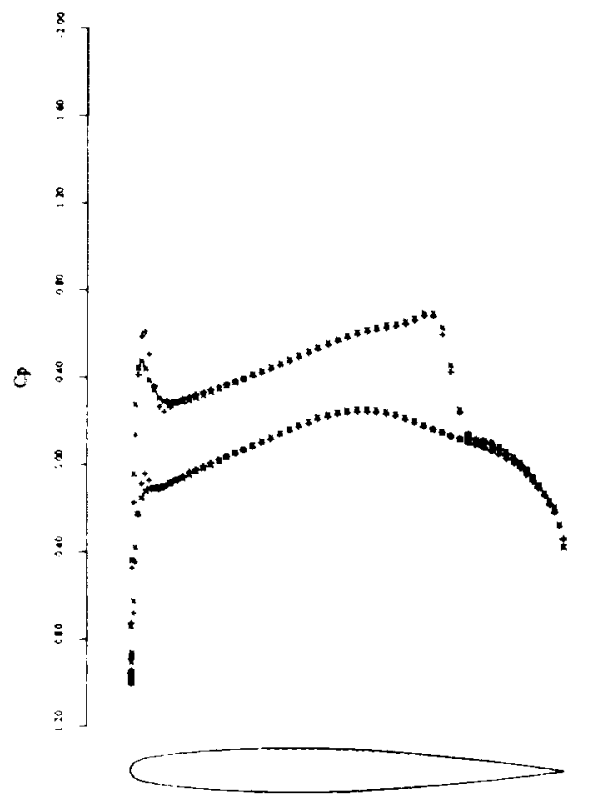

12a: span station $z=0.021$

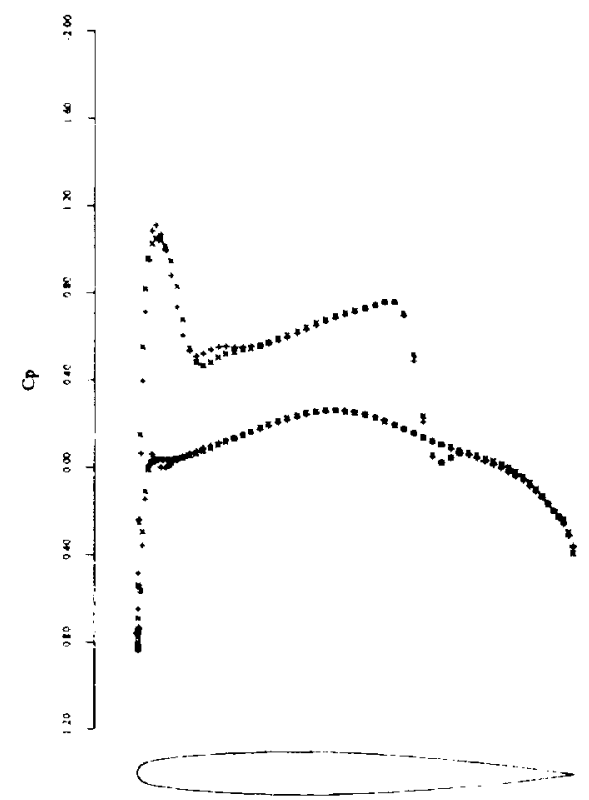

12b: span station $z=0.312$

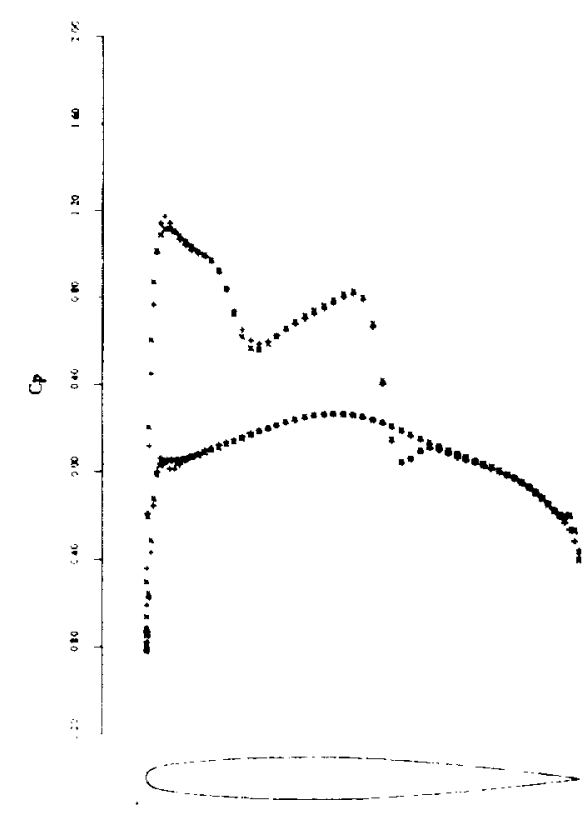

12c: span station $z=0.604$

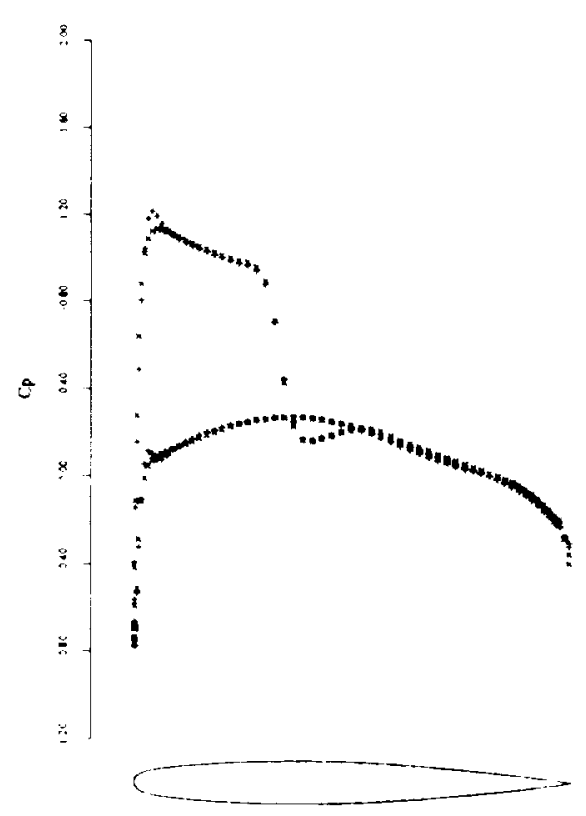

12d: span station $z=0.896$

Figure 12: Solution after 19 iterations for ONERA-M6 design.

$$
\begin{gathered}
M=0.84, C_{L}=0.1651, C_{D}=0.0078, a=3.060^{\circ} . \\
-\times \text { Initial Wing: NACA 0012. } \\
\cdots,+ \text { Target } C_{p}: \text { ONERA-M6. }
\end{gathered}
$$

150 Design Variables, $161 \times 41 \times 41$ Mesh, Euler Wing/Body Solution Inverse Design 


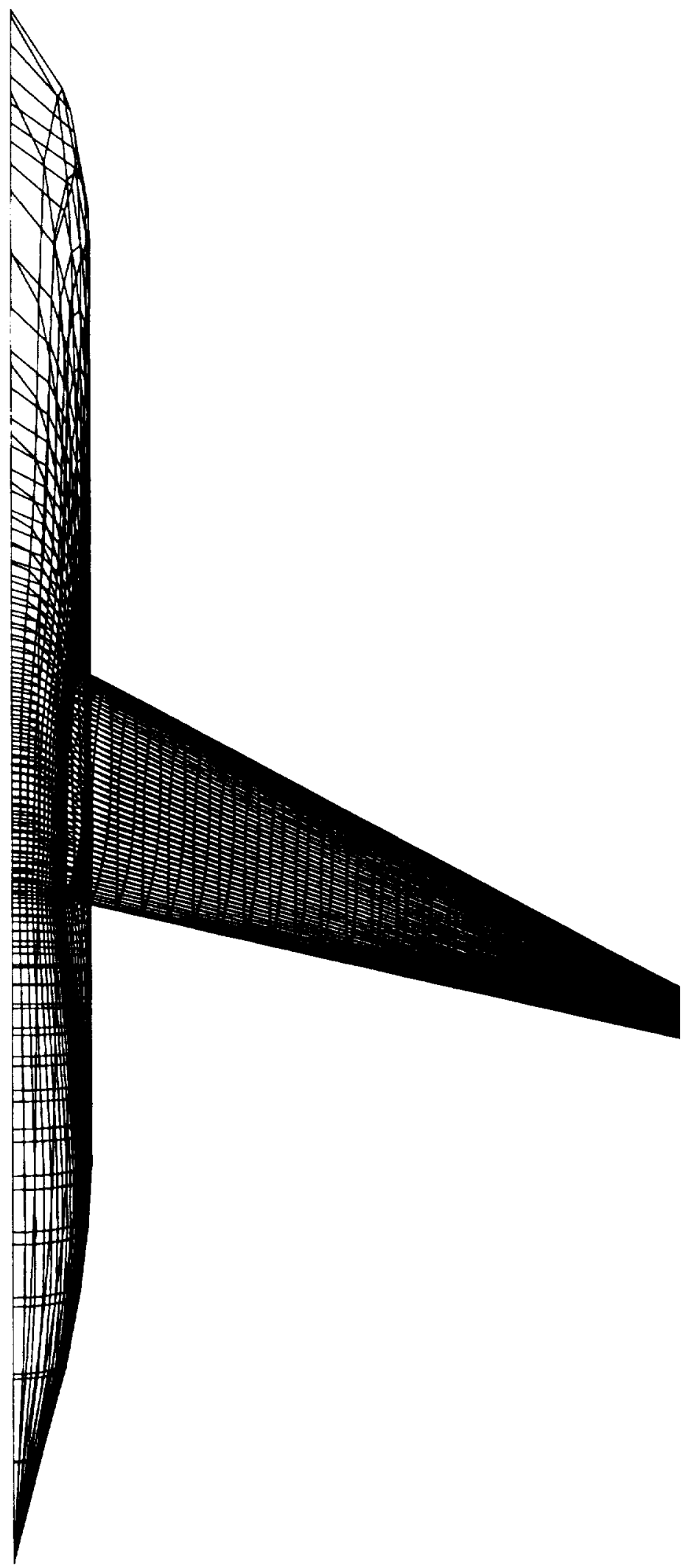

Figure 13: DC-9-30 Planform and Wing-Body mesh $161 \times 41 \times 41 \mathrm{C}-\mathrm{H}$ Topology 


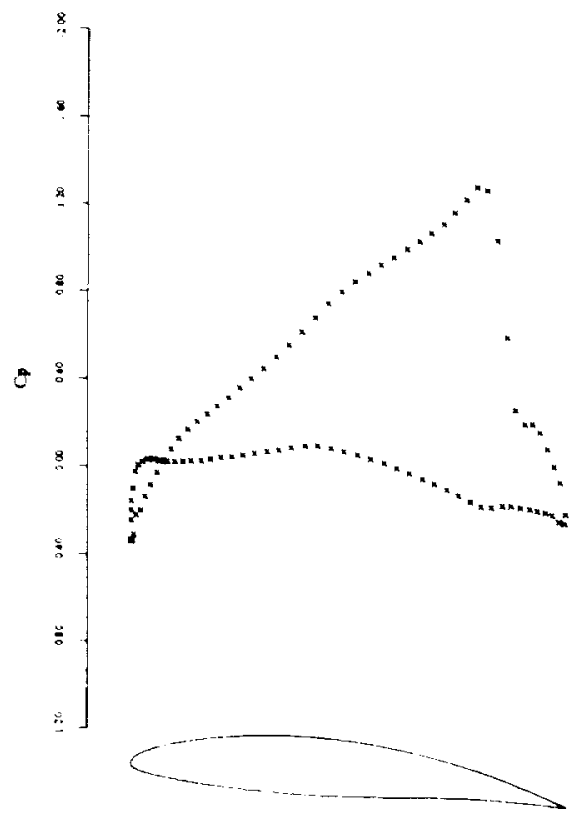

14a: span station $z=5.85 \mathrm{ft}$

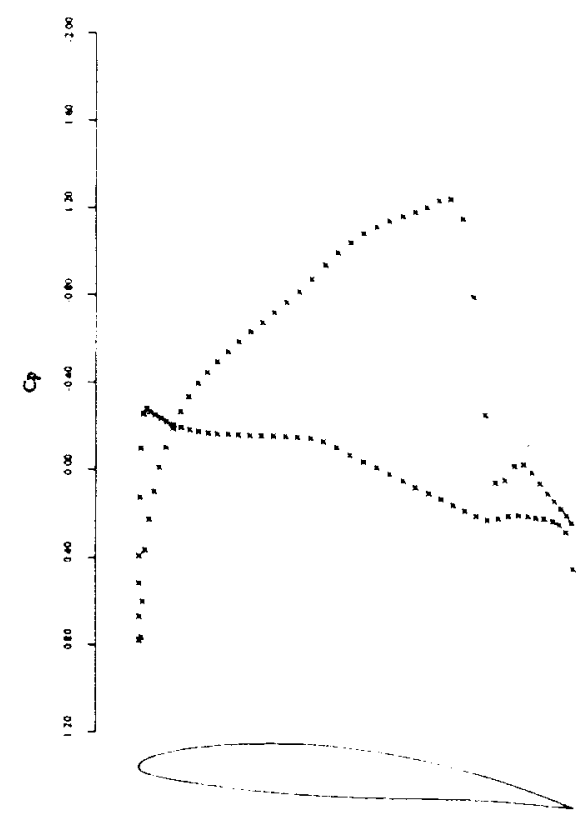

14b: span station $z=18.02 \mathrm{ft}$

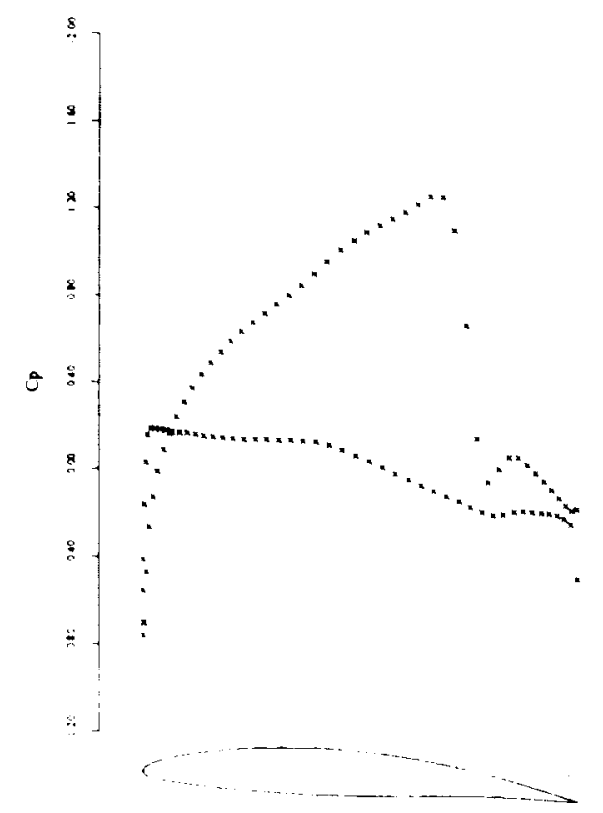

14c: span station $z=30.19 \mathrm{ft}$.

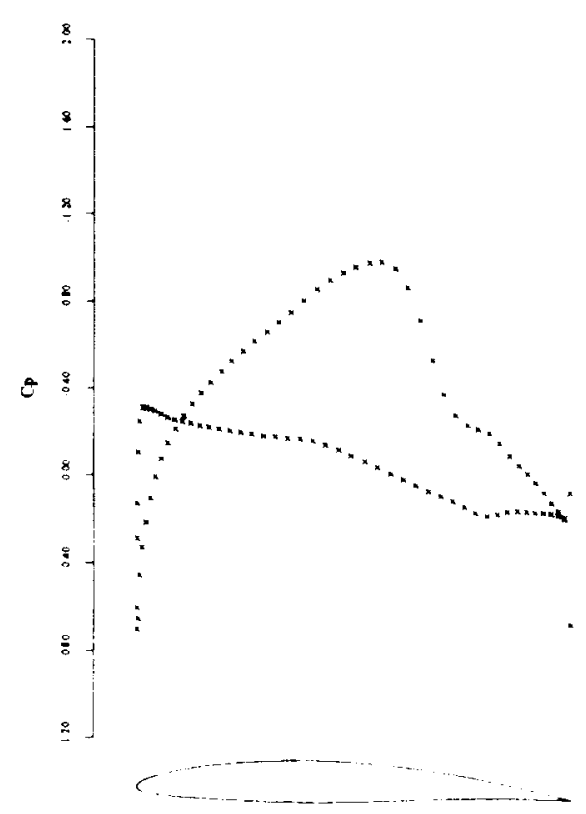

14d: span station $z=42.36 \mathrm{ft}$.

Figure 14: Initial condition for DC-9-30 design, $M=0.78$

$C_{L}=0.500, C_{L_{u}}=0.4535, C_{D_{u}}=0.0248, \alpha=-3.769^{\circ}$

117 Design Variables, $161 \times 41 \times 41$ Mesh, Euler Wing/Body Solution

Drag Minimization. 


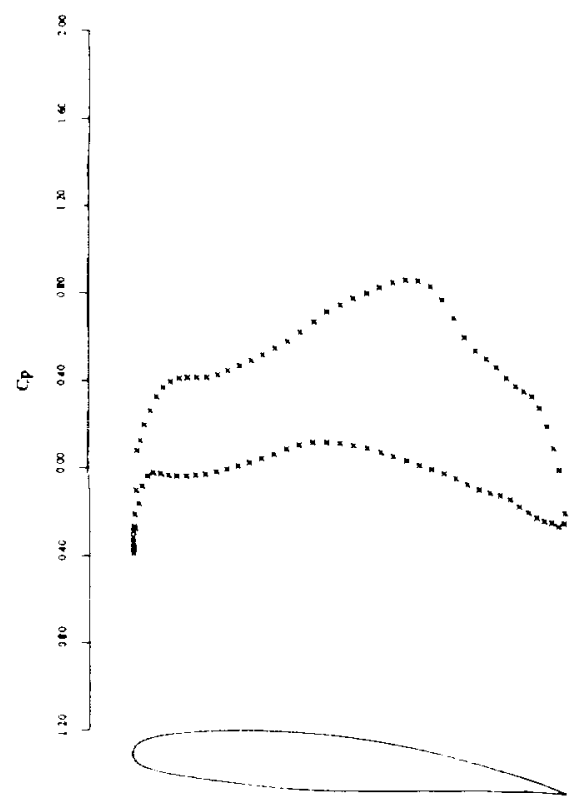

15a: span station $z=5.85 \mathrm{ft}$.

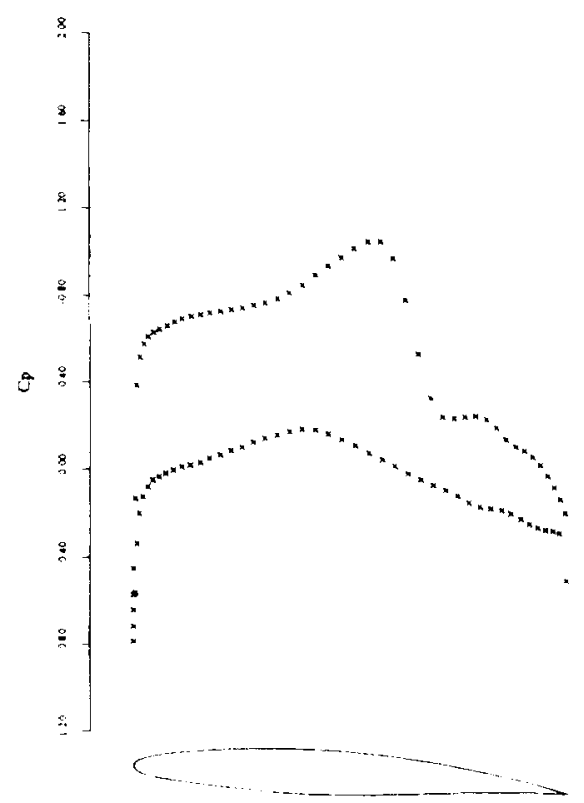

15c: span station $z=30.19 \mathrm{ft}$

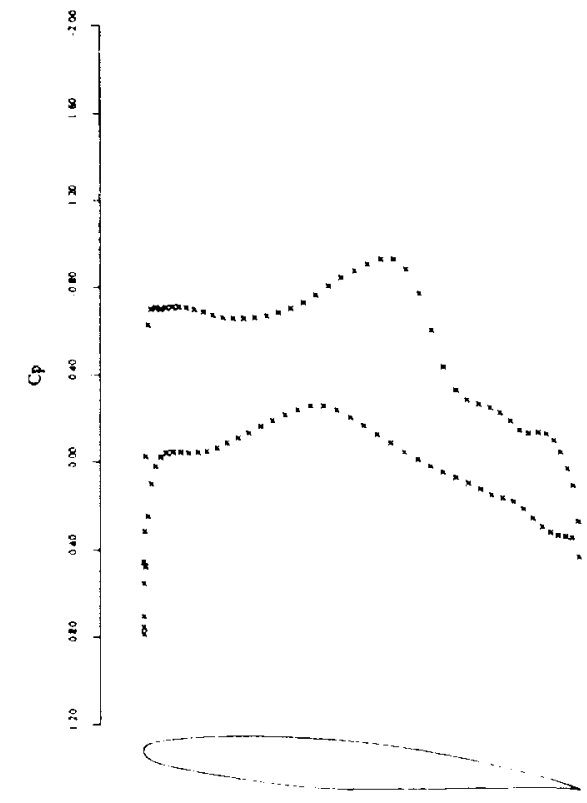

15b: span station $z=18.02 \mathrm{ft}$.

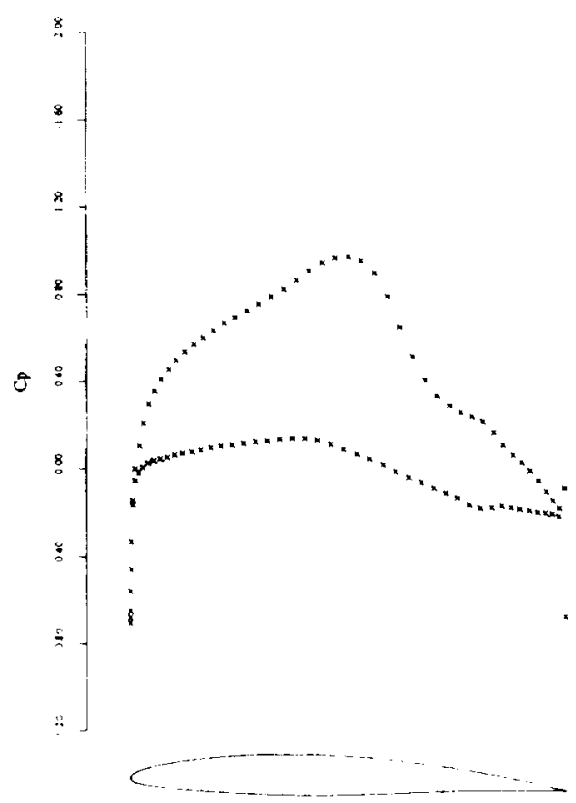

15d: span station $z=42.36 \mathrm{ft}$

Figure 15: DC-9-30 design after 16 iterations, $M=0.78$

$C_{L}=0.500, C_{L_{w}}=0.4478, C_{D_{w}}=0.0155, \alpha=-2.1776^{\circ}$

117 Design Variables, $161 \times 41 \times 41$ Mesh, Euler Wing/Body Solution

Drag Minimization. 


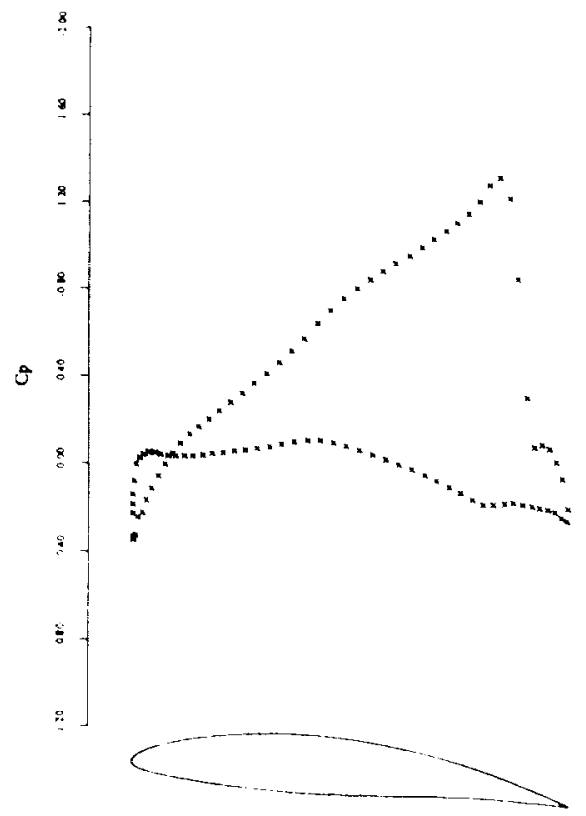

16a: span station $z=5.85 \mathrm{ft}$.

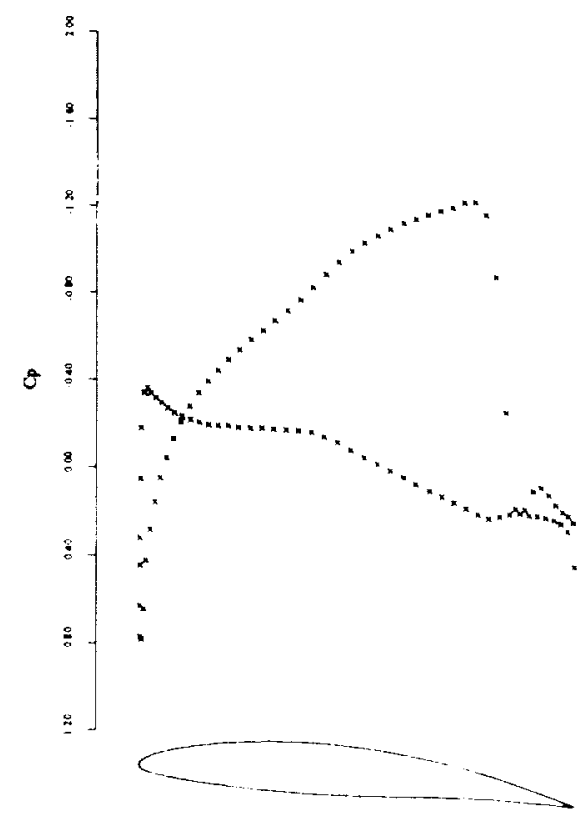

16b: span station $z=18.02 \mathrm{ft}$

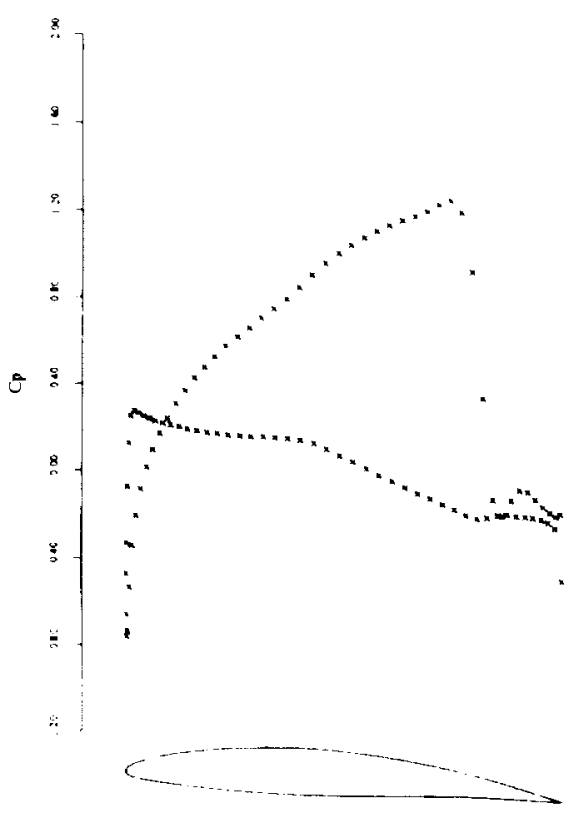

16c: span station $z=30.19 \mathrm{ft}$.

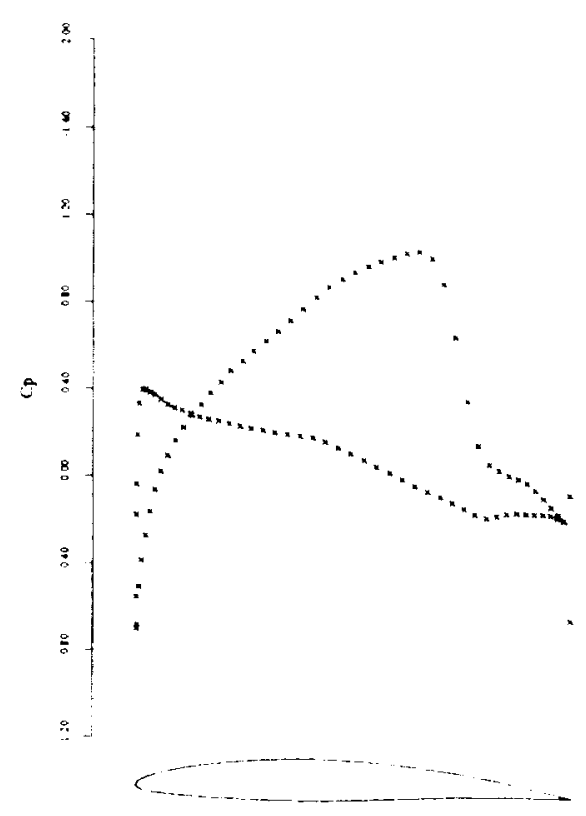

16d: span station $z=42.36 \mathrm{ft}$

Figure 16: Initial condition for DC-9-30 design, $M=0.80$

$C_{L}=0.500, C_{L_{w}}=0.4556, C_{D_{w}}=0.0315, \alpha=-3.960^{\circ}$

117 Design Variables, $161 \times 41 \times 41$ Mesh, Euler Wing/Body Solution

Drag Minimization. 


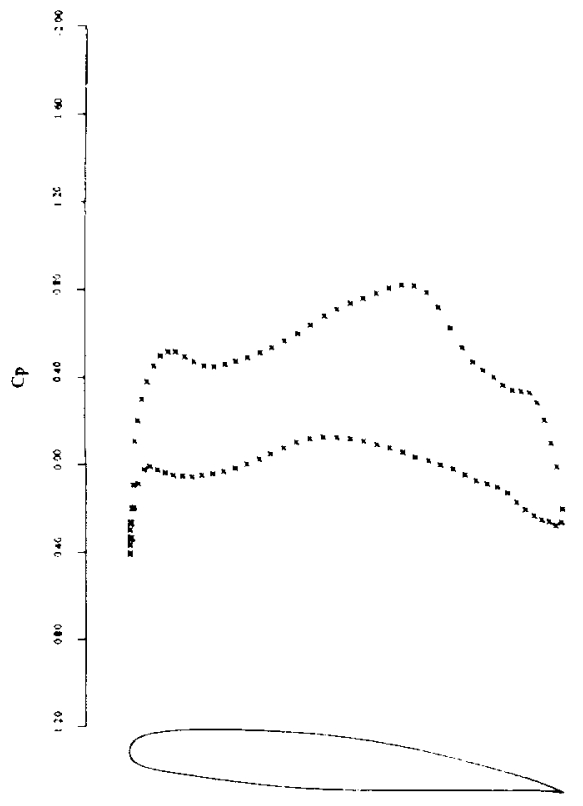

17a: span station $z=5.85 \mathrm{ft}$

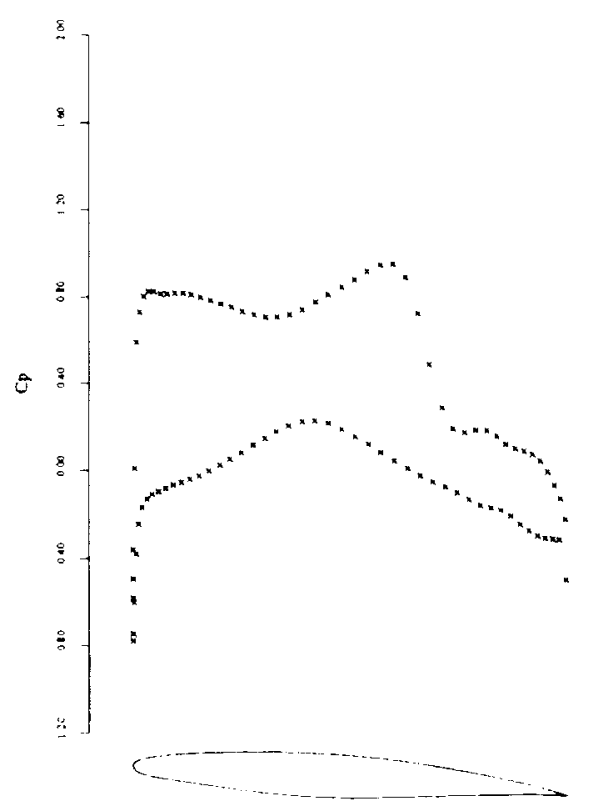

$17 \mathrm{c}$ : span station $z=30.19 \mathrm{ft}$

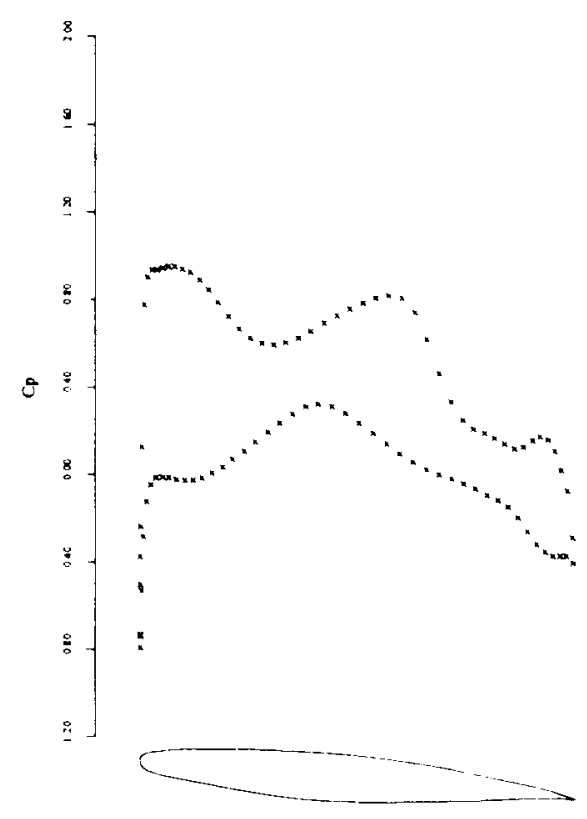

17b: span station $z=18.02 \mathrm{ft}$

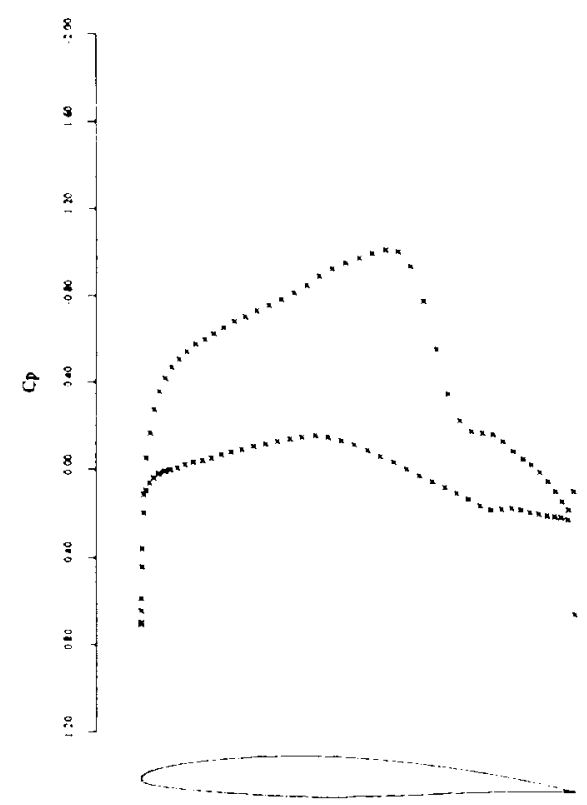

17d: span station $z=42.36 \mathrm{ft}$

Figure 17: DC-9-30 design after 15 iterations $M=0.80$

$C_{L}=0.500, C_{L_{w}}=0.4475, C_{D_{w}}=0.0167, \alpha=-1.8374^{\circ}$

117 Design Variables, $161 \times 41 \times 41$ Mesh, Euler Wing/Body Solution

Drag Minimization 
